\title{
OPTIMAL CONTROL OF THERMALLY CONVECTED FLUID FLOWS *
}

\section{K. ITO $^{\dagger}$ AND S.S. RAVINDRAN ${ }^{\dagger}$}

\begin{abstract}
We examine the optimal control of stationary thermally convected fluid flows from the theoretical and numerical point of view. We use thermal convection as control mechanism, that is, control is effected through the temperature on part of the boundary. Control problems are formulated as constrained minimization problem. Existence of optimal control is given and a first order necessary conditions of optimality from which optimal solutions can be obtained is established. We develop numerical methods to solve the necessary conditions of optimality and present computational results for control of cavity and channel type flows showing the feasibility of the proposed approach.
\end{abstract}

Key words. flow control, temperature control, optimization, Navier-Stokes equations, finite element methods

AMS subject classifications. 49J20, 76D05, 49B22, 49K20

\section{Contents}

\section{Introduction.}

1.1 The Governing Equations of A Thermally Convected Flow.

1.2 Statement of the Optimal Control Problem.

1.3 Notations

2. Weak Formulation.

2.1 Wellposedness

3. Existence of Optimal Controls and Necessary optimality condition.

4. Computational Methods.

4.1 Finite Element Approximation.

4.2 Newtons Method.

5. Computational Results.

5.1 Numerical Example 1.

5.2 Numerical Example 2.

6. Conclusion.

1. Introduction. The control of viscous flows for the purpose of achieving some desired objective is crucial to many technological and scientific applications. In the past, these control problems have been addressed either through expensive experimental processes or through the introduction of significant simplifications into the analyses used in the development of control mechanisms. Recently mathematicians and scientists have been able to address flow control problems in a systematic, rigorous manner and established a mathematical and numerical foundation for these problems; see [1-2], [4-5], [8-9]. [11], and [15-18].

* This work was supported in part by the Air Force Office of Scientific Research under grants AFOSR F49620-95-1-0437 and AFOSR F49620-95-1-0447.

$\dagger$ Center for Research in Scientific Computation, Department of Mathematics, North Carolina State University, Raleigh, NC 27695-8205 (kito@eos.ncsu.edu and ravi@eos.ncsu.edu). 
The control of vorticity has significant applications in science and engineering such as control of turbulence and control of crystal growth process. In this article we consider the minimization of vorticity in viscous incompressible thermally convected flows using boundary temperature as control mechanism.

We formulate the control problem as a constrained optimization problem for steady viscous incompressible thermally convected flow, namely that of computing a boundary temperature on a part of the boundary that minimizes the vorticity in the fluid. The constraint is the system of equations that represents steady viscous incompressible NavierStokes equations coupled with the energy equation. The choice for the cost is a quadratic functional involving the vorticity in the fluid so that a minimum of that functional corresponds to the minimum possible vorticity subject to the constraints. We then prove the existence of an optimal control and derive the first-order necessary conditions characterizing the control. Once the necessary optimality conditions are derived, we develop numerical methods to solve such conditions and present numerical results showing the feasibility of the approach for cavity and channel type flows.

1.1. The governing equations of a thermally convected flow. The class of thermally convective flow we consider is modelled by Boussinesq equations whose derivation is based on certain assumptions about the thermodynamics and the thermal effects on the flow. The first one is that variations in density is negligible except for the body force term $\rho \mathbf{g}$ in the momentum equations, where $\rho$ is the density and the vector $\mathbf{g}$ is the constant acceleration of gravity. We next assume that the density $\rho$ in the term $\rho \mathbf{g}$ can be given by $\rho=\rho_{0}\left[1-\beta\left(T-T_{0}\right)\right]$, where $T_{0}$ and $\rho_{0}$ are reference temperature and density, respectively, $\mathrm{T}$ is the absolute temperature and $\beta$ is the thermal expansion coefficient. Furthermore, we assume that in the energy equation, the dissipation of mechanical energy is negligible and the viscosity $\mu$, the heat conductivity $\kappa$, the thermal expansion coefficient $\beta$ and the specific heat at constant pressure $c_{p}$ are constant. Then under these assumptions the steady flow is governed by following equations:

$$
\begin{aligned}
& -\mu \Delta \mathbf{u}+\rho_{0}(\mathbf{u} \cdot \nabla) \mathbf{u}+\nabla p=\mathbf{g} \rho_{0}\left[1-\beta\left(T-T_{0}\right)\right] \text { in } \Omega, \\
& \nabla \cdot \mathbf{u}=0 \quad \text { in } \Omega, \\
& -\kappa \Delta T+\rho_{0} c_{p} \mathbf{u} \cdot \nabla T=0 \quad \text { in } \Omega,
\end{aligned}
$$

where $\Omega$ is a bounded open set and the heat source is assumed to be zero. If we assume there is a length scale $\ell$, a velocity scale $\mathbf{U}$ and a temperature scale $T_{1}-T_{0}$ in the flow, then one can define nondimensional Prandtl number $\operatorname{Pr}=\mu c_{p} / \kappa$, Grashof number $G r=$ $\beta \ell^{3} \rho_{0}^{2}|\mathbf{g}|\left(T_{1}-T_{0}\right) / \mu^{2}$ and Reynolds number $R e=\rho_{0} U \ell / \mu$. Next, if we nondimensionalize according to $\mathbf{x} \leftarrow \mathbf{x} / \ell, \mathbf{u} \leftarrow \mathbf{u} / \mathbf{U}, T \leftarrow\left(T-T_{0}\right) /\left(T_{1}-T_{0}\right)$, and $p \leftarrow(p-\mathbf{g} \cdot \mathbf{x}) /\left(\rho_{0} \mathbf{U}^{2}\right)$, we 
obtain the following nondimensional form of Boussinesq equations.

$$
\begin{aligned}
& -\frac{1}{R e} \Delta \mathbf{u}+(\mathbf{u} \cdot \nabla) \mathbf{u}+\nabla p+\frac{G r}{R e^{2}} T \mathbf{g}=\mathbf{0} \text { in } \Omega, \\
& \nabla \cdot \mathbf{u}=0 \quad \text { in } \Omega, \\
& -\frac{1}{R e P r} \Delta T+\mathbf{u} \cdot \nabla T=0 \text { in } \Omega,
\end{aligned}
$$

where $\mathbf{g}$ is now a unit vector in the direction of gravitational acceleration.

1.2. Statement of the optimal control problem. Let us next state the optimal control problem we consider

$$
\text { Minimize } \quad \mathcal{J}(\mathbf{u}, g)=\frac{1}{2} \int_{\Omega}|\nabla \times \mathbf{u}|^{2} d \Omega+\frac{\delta}{2} \int_{\Gamma_{1}}|g|^{2} d \Gamma
$$

subject to the state

$$
\left\{\begin{array}{l}
-\frac{1}{R e} \Delta \mathbf{u}+(\mathbf{u} \cdot \nabla) \mathbf{u}+\nabla p+\frac{G r}{R e^{2}} T \mathbf{g}=\mathbf{0} \text { in } \Omega, \\
\nabla \cdot \mathbf{u}=0 \text { in } \Omega, \\
-\frac{1}{\operatorname{RePr}} \Delta T+\mathbf{u} \cdot \nabla T=0 \text { in } \Omega .
\end{array}\right.
$$

with the boundary conditions as follows. Let $\Gamma=\Gamma_{0} \cup \Gamma_{1} \cup \Gamma_{2}$ where $\Gamma_{0}, \Gamma_{1}$ and $\Gamma_{2}$ are disjoint portions of the boundary $\Gamma$ of the domain $\Omega$.

$$
\left\{\begin{array}{l}
\mathbf{u}=\mathbf{u}_{0}, \quad T=T^{0} \text { on } \Gamma_{0} \\
\mathbf{u}=\mathbf{0}, \quad \frac{\partial T}{\partial \mathrm{n}}=h(g-T) \text { on } \Gamma_{1} \\
\mathbf{u}=\mathbf{0}, \quad T=T^{1} \text { on } \Gamma_{2},
\end{array}\right.
$$

where $\mathbf{u}_{0}, T^{0}$ and $T^{1}$ are given on the boundary and $g$ is a temperature control by the radiational heating or cooling. In the cost functional $\mathcal{J}$, the term $\int_{\Omega}|\nabla \times \mathbf{u}|^{2} d \Omega$ is a measure of vorticity $\boldsymbol{\omega}=\nabla \times \mathbf{u}$ in the flow, the term $\int_{\Gamma_{1}}|g|^{2} d \Gamma$ is the measure of the magnitude of the control which is also required for the rigorous mathematical analysis of the control problem and the penalizing parameter $\delta$ adjusts the size of the terms in the cost. The flow quantities $\mathbf{u}, \mathrm{T}$ and $\mathrm{p}$ denote as usual the velocity, temperature and pressure, respectively.

The outline of the paper is as follows. In $\$ 2$, we give a variational formulation of the state equations and study their wellposedness. We believe it is new since it deals with nonhomogeneous boundary conditions. In $\S 3$ the existence of optimal solutions and first order optimality conditions for optimal control problems are established. $\$ 4$ deals with computational methods to solve the necessary conditions of optimality. Finally, in $\S 5$, we present numerical results for control of cavity and channel flows using boundary temperature controls. 
1.3. Notations. Throughout, $C$ or $C_{i}$ (where $i$ is any subscript) denotes a constant depending only the domain $\Omega$ which is assumed to be a bounded set in $\mathbb{R}^{2}$ with smooth boundary $\Gamma$. We denote by $L^{2}(\Omega)$ the collection of square-integrable functions defined on $\Omega$. Let

$$
\begin{gathered}
H^{1}(\Omega)=\left\{v \in L^{2}(\Omega): \frac{\partial v}{\partial x_{i}} \in L^{2}(\Omega) \text { for } i=1,2\right\}, \quad H_{0}^{1}(\Omega)=\left\{v \in H^{1}:\left.v\right|_{\Gamma}=0\right\}, \\
L_{0}^{2}(\Omega)=\left\{q \in L^{2}(\Omega): \int_{\Omega} q d \Omega=0\right\}
\end{gathered}
$$

and $H^{m}(\Omega)=\left\{v \in L^{2}(\Omega): \frac{\partial|\alpha|_{v}}{\partial x_{1}^{\alpha_{1} \partial x_{2}}} \in L^{2}(\Omega)\right.$, for all $\alpha=\left(\alpha_{1}, \alpha_{2}\right)$ with $\left.|\alpha| \leq m\right\}$. Vector-valued counterparts of these spaces are denoted by bold-face symbols, e.g., $\mathbf{H}^{1}(\Omega)=$ $\left[H^{1}(\Omega)\right]^{2}$. The trace spaces $H^{r}(\Gamma)(r>0)$ are the restriction to the boundary of $H^{r+1 / 2}(\Omega)$. We denote the norms and inner products for $H^{s}(\Omega)$ or $\mathbf{H}^{s}(\Omega)$ by $\|\cdot\|_{s}$ and $(\cdot, \cdot)_{s}$, respectively. The $L^{2}(\Omega)$ or $\mathrm{L}^{2}(\Omega)$ inner product is denoted by $(\cdot, \cdot)$. We denote the norms and inner products for $H^{r}(\Gamma)$ or $\mathbf{H}^{r}(\Gamma)$ by $\|\cdot\|_{r, \Gamma}$ and $(\cdot, \cdot)_{r, \Gamma}$, respectively. The $L^{2}(\Gamma)$ or $\mathbf{L}^{2}(\Gamma)$ inner product is denoted by $(\cdot, \cdot)_{\Gamma}$.

Let $\mathbf{V}_{0}$ be the divergence free subspace of $\mathbf{H}_{0}^{1}$ defined by

$$
\mathbf{V}_{0}=\left\{\mathbf{v} \in \mathbf{H}_{0}^{1}(\Omega): \quad \nabla \cdot \mathbf{v}=0\right\}
$$

and $\mathbf{H}_{0}$ is the completion of $\mathbf{V}_{0}$ with respect to $\mathbf{L}^{2}(\Omega)$ norm and is given by

$$
\mathbf{H}_{0}=\left\{\mathbf{v} \in \mathbf{L}^{2}(\Omega): \nabla \cdot \mathbf{v}=0 \text {, and }\left.\mathbf{v} \cdot \mathbf{n}\right|_{\Gamma}=0\right\} .
$$

The space $\mathbf{H}_{0}$ is equipped with the norm $\|\cdot\|_{0}$ and $\mathbf{V}_{0}$ is equipped with $|\mathbf{u}|_{1}=\|\nabla \mathbf{u}\|_{0}$. Let $V_{1}$ be the subspace of $H^{1}(\Omega)$ defined by

$$
V_{1}=\left\{\psi \in H^{1}(\Omega): \psi=0 \text { on } \Gamma_{0} \cup \Gamma_{2}\right\}
$$

and set $\mathbf{V}=V_{0} \times V_{1}$. Let $\mathbf{V}_{0}^{*}$ and $V_{1}^{*}$ be the strong dual spaces of $\mathbf{V}_{0}$ and $V_{1}$, respectively, and $\langle\cdot, \cdot\rangle$ denote the dual product on either $\mathbf{V}_{0}^{*} \times \mathbf{V}_{0}$ or $V_{1}^{*} \times V_{1}$. Throughout the mathematical discussions, for the sake of convenience we set $\widehat{\nu}=\frac{1}{R e}, \widehat{\kappa}=\frac{1}{R e P r}$ and $\widehat{\alpha}=\frac{G r}{R e^{2}}$ which are not to be confused with the physical quantities such as kinematic viscosity and conductivity.

We define the following bilinear and trilinear forms

$$
\begin{gathered}
a_{0}(\mathbf{u}, \mathbf{v})=\int_{\Omega} \hat{\nu}(\nabla \mathbf{u}):(\nabla \mathbf{v}) d \Omega \quad \forall \mathbf{u}, \mathbf{v} \in \mathbf{H}^{1}(\Omega), \\
a_{1}(T, \psi)=\int_{\Omega} \widehat{\kappa} \nabla T \cdot \nabla \psi d \Omega \quad \forall T, \psi \in H^{1}(\Omega), \\
c(\mathbf{u}, q)=-\int_{\Omega} q \nabla \cdot \mathbf{u} d \Omega \quad \forall \mathbf{u} \in \mathbf{H}^{1}(\Omega), \forall q \in L^{2}(\Omega),
\end{gathered}
$$




$$
b_{0}(\mathbf{u}, \mathbf{v}, \mathbf{w})=\int_{\Omega}(\mathbf{u} \cdot \nabla) \mathbf{v} \cdot \mathbf{w} d \Omega \quad \forall \mathbf{u}, \mathbf{v}, \mathbf{w} \in \mathbf{H}^{1}(\Omega)
$$

and

$$
b_{1}(\mathbf{u}, T, \psi)=\int_{\Omega} \mathbf{u} \cdot \nabla T, \psi d \Omega \quad \forall \mathbf{u} \in \mathbf{H}^{1}(\Omega), \forall T, \psi \in H^{1}(\Omega) .
$$

We have the coercivity relations associated with $a_{0}(\cdot, \cdot)$ and $a_{1}(\cdot, \cdot)$ :

$$
a_{0}(\mathbf{u}, \mathbf{u})=\widehat{\nu}\|\nabla \mathbf{u}\|_{0}^{2} \geq C_{1}\|\mathbf{u}\|_{1}^{2} \quad \forall \mathbf{u} \in \mathbf{H}_{0}^{1}(\Omega)
$$

and

$$
a_{1}(T, T)=\widehat{\kappa}\|\nabla T\|_{0}^{2} \geq C_{2}\|T\|_{1}^{2} \quad \forall T \in H^{1}(\Omega) \cap V_{1}
$$

which are a direct consequence of Poincaré inequality.

2. Weak Formulation. In this section we discuss the weak variational formulation of the Boussinesq system (1.2) and establish the existence of weak solutions.

It follows from the Hopf extension (see [10]) that for each $\epsilon>0$, there exists a function $\overline{\mathbf{u}} \in \mathbf{H}^{1}(\Omega)$ such that $\nabla \cdot \overline{\mathbf{u}}=0$ and $\left.\overline{\mathbf{u}}\right|_{\Gamma_{0}}=\mathbf{u}_{0},\left.\overline{\mathbf{u}}\right|_{\Gamma_{1} \cup \Gamma_{2}}=\mathbf{0}$ and

$$
\left|b_{0}(\mathbf{v}, \overline{\mathbf{u}}, \mathbf{v})\right| \leq \epsilon|\mathbf{v}|_{1}^{2} \forall \mathbf{v} \in \mathbf{V}_{0}
$$

provided that the boundary data $\mathbf{u}_{0} \in \mathbf{H}^{\frac{1}{2}}(\Gamma)$ satisfies $\left(\mathbf{n} \cdot \mathbf{u}_{0}, 1\right)_{\Gamma_{0}}=0$. In the sequel we will take $\epsilon=\frac{\hat{\nu}}{2}$. Let $\bar{T} \in H^{1}(\Omega)$ be a function such that $\left.\bar{T}\right|_{\Gamma_{0}}=T^{0}$ and $\left.\bar{T}\right|_{\Gamma_{2}}=T^{1}$. Then any function $(\mathbf{u}, T) \in \mathbf{H}^{1}(\Omega) \times H^{1}(\Omega)$ satisfying the inhomogeneous boundary condition (1.3) and $\nabla \cdot \mathbf{u}=0$ can be represented by

$$
(\mathbf{u}, T)=(\mathbf{w}, \theta)+(\overline{\mathbf{u}}, \bar{T}) \text { where }(\mathbf{w}, \theta) \in \mathbf{V}=\mathbf{V}_{0} \times V .
$$

We then obtain a weak variational form of $(1.2)$. For $(\mathbf{u}, T) \in \mathbf{V}+(\overline{\mathbf{u}}, \bar{T})$,

$$
\begin{aligned}
& a_{0}(\mathbf{u}, \mathbf{v})+b_{0}(\mathbf{u}, \mathbf{u}, \mathbf{v})+\widehat{\alpha}(T \mathbf{g}, \mathbf{v})=\mathbf{0} \forall \mathbf{v} \in \mathbf{V}_{0} \\
& a_{1}(T, \psi)+b_{1}(\mathbf{u}, T, \psi)+\widehat{\kappa} h(T-g, \psi)_{\Gamma_{1}}=0 \quad \forall \quad \psi \in V_{1} .
\end{aligned}
$$

A solution $(\mathbf{u}, T) \in \mathbf{V}+(\overline{\mathbf{u}}, \bar{T})$ is called a weak solution of (1.2) if equation (2.1) is satisfied.

Regarding the bilinear form $b_{0}(\cdot, \cdot, \cdot)$, we have the following results.

Lemma 2.1. For $\mathbf{u}, \mathbf{v}, \mathbf{w} \in \mathbf{H}^{1}(\Omega)$, the trilinear form $b_{0}(\cdot, \cdot, \cdot)$ satisfies

$$
\begin{aligned}
& \left|b_{0}(\mathbf{u}, \mathbf{v}, \mathbf{w})\right| \leq C_{3}\|\mathbf{u}\|_{1}\|\mathbf{v}\|_{1}\|\mathbf{w}\|_{1} \\
& \text { and } b_{0}(\mathbf{u}, \mathbf{v}, \mathbf{w})+b_{0}(\mathbf{u}, \mathbf{w}, \mathbf{v})=0 \text { for } \nabla \cdot \mathbf{u}=0 \text { and } \mathbf{w} \in \mathbf{V}_{0} .
\end{aligned}
$$


Proof. The first inequality follows from the Holders inequality. We obtain

$$
\left|b_{0}(\mathbf{u}, \mathbf{v}, \mathbf{w})\right| \leq\left\|\left.\left.\mathbf{u}\right|_{L^{4}}|| \nabla \mathbf{v}\right|_{L^{2}}\right\| \mathbf{w}\left\|_{L^{4}} \leq C_{3}\right\| \mathbf{u}\left\|_{1}\right\| \mathbf{v}\left\|_{1}\right\| \mathbf{w} \|_{1} .
$$

The second result follows from Green's formula

$$
b_{0}(\mathbf{u}, \mathbf{v}, \mathbf{w})+b_{0}(\mathbf{u}, \mathbf{w}, \mathbf{v})=(\mathbf{u}, \nabla(\mathbf{v} \cdot \mathbf{w}))=(\mathbf{n} \cdot \mathbf{u}, \mathbf{v} \cdot \mathbf{w})_{\Gamma}
$$

provided that $\nabla \cdot \mathbf{u}=0$ and $\mathbf{v} \in \mathbf{H}^{1}(\Omega)$.

It follows from the proof of Lemma 2.1 that

$$
b_{0}(\mathbf{u}, \mathbf{v}, \mathbf{v})=0 \quad \text { for } \mathbf{v} \in \mathbf{V}_{0} \text { and } \nabla \cdot \mathbf{u}=0
$$

and

$$
b_{1}(\mathbf{u}, T, \psi)+b_{1}(\mathbf{u}, \psi, T)=0
$$

for $\mathbf{u} \in \mathbf{V}_{0}+\overline{\mathbf{u}}$ and $\psi \in V_{1}$.

2.1. Wellposedness. In this section we prove the existence of a weak solution to (2.1). Let $\mathbf{Z}=\mathbf{V}+(\overline{\mathbf{u}}, \bar{T})$.

Theorem 2.2. Given $g \in L^{2}\left(\Gamma_{1}\right)$ there exists a weak solution $(\mathbf{u}, T) \in \mathbf{Z}$ to (2.1) and

$$
\|(\mathbf{u}, T)\|_{1} \leq C\left(\|g\|_{0, \Gamma_{1}}+\|\bar{T}\|_{1}\right) .
$$

Moreover, if $g(x), T^{0}(\mathbf{x})$ and $T^{1}(\mathbf{x})$ are bounded below by $\bar{T}_{1}$ and bounded above by $\bar{T}_{2}$ almost everywhere then $\bar{T}_{1} \leq T(x) \leq \bar{T}_{2}$ almost everywhere in $\Omega$ for every solution.

Proof. Step I (Existence): We show that (2.1) has a solution $(\mathbf{u}, T) \in \mathbf{Z}$. Given $\widehat{\mathbf{u}} \in \mathbf{V}_{0}+\overline{\mathbf{u}}$ and $(\mathbf{w}, \theta) \in \mathbf{V}$, we define linear equations by

$$
\begin{gathered}
a_{0}(\mathbf{u}, \mathbf{v})+b_{0}(\widehat{\mathbf{u}}, \mathbf{w}, \mathbf{v})+b_{0}(\mathbf{u}, \overline{\mathbf{u}}, \mathbf{v})+(\widehat{\alpha} T \mathbf{g}, \mathbf{v})=0 \quad \text { for } \mathbf{v} \in \mathbf{V}_{0}, \\
a_{1}(T, \psi)+b_{1}(\widehat{\mathbf{u}}, T, \psi)+\widehat{\kappa} h(T-g, \psi)_{\Gamma_{1}}=0 \quad \text { for } \psi \in V_{1},
\end{gathered}
$$

where $\mathbf{u}=\mathbf{w}+\overline{\mathbf{u}}, \quad \widehat{\mathbf{u}}=\widehat{\mathbf{w}}+\overline{\mathbf{u}}$, and $T=\theta+\bar{T}$. First, we show that (2.6) has a unique solution $(\mathbf{w}, \theta) \in \mathbf{V}$. Then, we show that the solution map $S$ on $\mathbf{V}_{0}+\overline{\mathbf{u}}$ defined by $S(\widehat{\mathbf{u}})=\mathbf{u}$, where $(\mathbf{u}, T) \in \mathbf{Z}$ is the unique solution to (2.6), has a fixed point by Schäuder fixed point theorem. The fixed point $\mathbf{u} \in \mathbf{V}_{0}+\overline{\mathbf{u}}$ and the corresponding solution $T \in V_{1}+\bar{T}$ define a solution to (2.1).

We first note, from Lemma 2.1 and $(2.5)$, that the bilinear form $\sigma_{1}(\cdot, \cdot)$ defined by

$$
\sigma_{1}(\cdot, \cdot)=a_{1}(\cdot, \cdot)+b_{1}(\widehat{\mathbf{u}}, \cdot, \cdot)+\widehat{\kappa} h(\cdot, \cdot)_{\Gamma_{1}}
$$


on $V_{1} \times V_{1}$ is bounded and $V_{1}$-coercive. It thus follows from Lax-Milgram theorem that the equation

$$
\sigma_{1}(\theta, \psi)=\widehat{\kappa} h(g, \psi)_{\Gamma_{1}}-a_{1}(\bar{T}, \psi)-b_{1}(\widehat{\mathbf{u}}, \bar{T}, \psi)
$$

for $\psi \in V_{1}$ has a unique solution $\theta \in V_{1}$ and $T=\theta+\bar{T}$ satisfies (2.6b).

Setting $\psi=\sup \left(0, T-\bar{T}_{2}\right) \in V_{1}$ in $(2.6 \mathrm{~b})$, we have

$$
a_{1}(T, \psi)+b_{1}(\widehat{\mathbf{u}}, T, \psi)+\widehat{\kappa} h\left(T-\bar{T}_{2}, \psi\right)_{\Gamma_{1}}=\widehat{\kappa} h\left(g-\bar{T}_{2}, \psi\right)_{\Gamma_{1}} .
$$

It follows from $(2.5)$ that $b_{1}(\widehat{\mathbf{u}}, \psi, \psi)=0$ and thus

$$
a_{1}(\psi, \psi)+\frac{1}{2} \widehat{\kappa} h\|\psi\|_{0, \Gamma_{1}}^{2} \leq \frac{1}{2} \widehat{\kappa} h\left\|g-\bar{T}_{2}\right\|_{0, \Gamma_{1}}^{2} .
$$

This implies

$$
\|\psi\|_{0} \leq C_{4}\left\|g-\bar{T}_{2}\right\|_{0, \Gamma_{1}} .
$$

Similarly, letting $\eta=\inf \left(0, T-\bar{T}_{1}\right) \in V_{1}$ we obtain

$$
\|\eta\|_{0} \leq C_{5}\left\|g-\bar{T}_{1}\right\|_{0, \Gamma_{1}} .
$$

From the definition of $\eta$ and $\psi$, it follows that $\|T\|_{0} \leq C_{6}$ which is independent of $\widehat{\mathbf{u}} \in \mathbf{V}_{0}+\overline{\mathbf{u}}$.

Next, we define the bilinear form $\sigma_{0}(\cdot, \cdot)$ on $\mathbf{V}_{0} \times \mathbf{V}_{0}$ by

$$
\sigma_{0}(\mathbf{w}, \mathbf{v})=a_{0}(\mathbf{w}, \mathbf{v})+b_{0}(\hat{\mathbf{u}}, \mathbf{w}, \mathbf{v})+b_{0}(\mathbf{w}, \overline{\mathbf{u}}, \mathbf{v}) .
$$

It then follows from Lemma 2.1, (2.4) and the inequality

$$
\left|b_{0}(\mathbf{v}, \overline{\mathbf{u}}, \mathbf{v})\right| \leq \frac{1}{2} a_{0}(\mathbf{v}, \mathbf{v})
$$

that $\sigma_{0}(\cdot, \cdot)$ is bounded and $V_{0}$-coercive. Thus, by Lax-Milgram theorem, the equation

$$
\sigma_{0}(\mathbf{w}, \mathbf{v})=-(\hat{\alpha} T \mathbf{g}, \mathbf{v})-b_{0}(\overline{\mathbf{u}}, \overline{\mathbf{u}}, \mathbf{v})-a_{0}(\overline{\mathbf{u}}, \mathbf{v}),
$$

for $\mathbf{v} \in \mathbf{V}_{0}$, has a unique solution $\mathbf{w} \in \mathbf{V}_{0}$ and $\mathbf{u}=\mathbf{w}+\overline{\mathbf{u}}$ satisfies (2.6a). Setting $\mathbf{v}=\mathbf{w}$ in (2.6a) and using the estimate $\|T\|_{0} \leq C_{6}$, we get

$$
|\mathbf{w}|_{1} \leq \frac{2 C_{7}}{\widehat{\nu}}\|T\|_{0}++\frac{2 C_{3}}{\widehat{\nu}}\left(\|\overline{\mathbf{u}}\|_{1}^{2}+\|\overline{\mathbf{u}}\|_{1}\right) \leq \gamma
$$

where $\|\mathbf{v}\|_{0} \leq C_{7}|\mathbf{v}|_{1}, \mathbf{v} \in \mathbf{V}_{0}$. Let $\mathbf{\Sigma}$ be a closed convex subspace of $\mathbf{H}^{1}(\Omega)$, defined by

$$
\Sigma=\left\{\mathbf{u}=\mathbf{w}+\overline{\mathbf{u}}: \mathbf{w} \in \mathbf{V}_{0} \text { satisfying }|\mathbf{w}|_{1} \leq \gamma\right\} .
$$

Then it follows from (2.7) that $S$ maps from $\boldsymbol{\Sigma}$ into $\boldsymbol{\Sigma}$. Moreover, the solution map $S$ is compact. In fact, if $\widehat{\mathbf{w}}_{k}$ converges weakly to $\widehat{\mathbf{w}}$ in $\mathbf{V}_{0}$ then $\left\|\widehat{\mathbf{u}}_{k}-\widehat{\mathbf{u}}\right\|_{L^{4}} \rightarrow 0$, since $H^{1}(\Omega)$ 
is compactly embedded into $L^{4}(\Omega)$. Let $\left(\mathbf{u}_{k}, T_{k}\right) \in \mathbf{Z}$ and $(\mathbf{u}, T) \in \mathbf{Z}$ be the corresponding solution of (2.6), respectively to $\widehat{\mathbf{u}}_{k}$ and $\widehat{\mathbf{u}}$. Then we have

$$
a_{1}\left(T_{k}-T, \psi\right)+b_{1}\left(\widehat{\mathbf{u}}_{k}-\widehat{\mathbf{u}}, T, \psi\right)+b_{1}\left(\widehat{\mathbf{u}}_{k}, T_{k}-T, \psi\right)+\widehat{\kappa} h\left(T_{k}-T, \psi\right)_{\Gamma_{1}}=0
$$

for $\psi \in V_{1}$. Setting $\psi=T_{k}-T$, we have from Lemma 2.1 and $(2.5)$ that

$$
\left\|T_{k}-T\right\|_{1} \leq C_{8}\left\|\widehat{\mathbf{u}}_{k}-\widehat{\mathbf{u}}\right\|_{L^{4}}\|T\|_{1}
$$

which implies $\left\|T_{k}-T\right\|_{1} \rightarrow 0$. Similarly, we have

$$
\widehat{\nu}\left|\mathbf{u}_{k}-\mathbf{u}\right|_{1} \leq C_{9}\left\|\widehat{\mathbf{u}}_{k}-\widehat{\mathbf{u}}\right\|_{L^{4}}|\mathbf{w}|_{1}+\widehat{\alpha} C_{10}\left\|T_{k}-T\right\|_{0}
$$

and thus $\left|\mathbf{u}_{k}-\mathbf{u}\right|_{1} \rightarrow 0$. Now, by Schäuder fixed point theorem (see [20]) there exists at least one solution to $(2.6)$.

Let us next derive the appriori estimate. Setting $\psi=\theta$ in $(2.6 \mathrm{~b})$ we obtain

$$
a_{1}(\theta+\bar{T}, \theta)+b_{1}(\widehat{\mathbf{u}}, \theta+\bar{T}, \theta)+\widehat{\kappa} h(\theta+\bar{T}-g, \theta)_{\Gamma_{1}}=0 .
$$

Equivalently,

$$
a_{1}(\theta, \theta)+b_{1}(\widehat{\mathbf{u}}, \theta, \theta)+\widehat{\kappa} h(\theta, \theta)_{\Gamma_{1}}=-a_{1}(\bar{T}, \theta)-b_{1}(\widehat{\mathbf{u}}, \bar{T}, \theta)-\widehat{\kappa} h(\bar{T}-g, \theta)_{\Gamma_{1}} .
$$

Then using the coercivity and continuity properties of $a_{1}(\cdot, \cdot)$ and $b_{1}(\cdot, \cdot, \cdot)$ and the antisymmetry property of $b_{1}(\cdot, \cdot, \cdot)$, it follows that

$$
\|\theta\|_{1} \leq C_{11}\left(\|\bar{T}\|_{1}+\|g\|_{0, \Gamma_{1}}\right)
$$

for some constant $C_{11}$ independent of $\widehat{\mathbf{u}}$. From (2.7)-(2.8), we obtain the appriori estimate

$$
\|(\mathbf{u}, T)\|_{1} \leq\|(\mathbf{w}, \theta)\|_{1}+\|(\overline{\mathbf{u}}, \bar{T})\|_{1} \leq C\left(\|g\|_{0, \Gamma_{1}}+\|\bar{T}\|_{1}\right)
$$

for some constant $\mathrm{C}$.

Step II ( $L^{\infty}$ estimate): We show that if $\bar{T}_{1} \leq g \leq \bar{T}_{2}$ then

$$
\bar{T}_{1} \leq T \leq \bar{T}_{2} \quad \text { almost everywhere } x \in \Omega .
$$

for every solution $(\mathbf{u}, T) \in \mathbf{Z}$ to $(2.1)$. In fact, letting $\psi=\inf \left(0, T-\bar{T}_{1}\right)$ in the second equation of (2.1) and using the same arguments as above, we obtain

$$
a_{1}(\psi, \psi)+\hat{\kappa} h(T-g, \psi)_{\Gamma_{1}}=0
$$

where

$$
(T-g) \psi=\left(T-\bar{T}_{1}-\left(g-\bar{T}_{1}\right)\right) \psi \geq|\psi|^{2} \text { on } \Gamma_{1} .
$$

Thus, we obtain $\|\psi\|_{1}^{2}=0$ which implies $\psi=0$ and hence $T \geq \bar{T}_{1}$. Similarly, one can prove that $T \leq \bar{T}_{2}$, choosing the test function $\psi=\sup \left(0, T-\bar{T}_{2}\right)$. 
We also have the uniqueness of solutions under the smallness assumption on $\overline{\mathbf{u}}$ and $\bar{T}_{1}-\bar{T}_{2}$.

Theorem 2.3. If $g(x), T^{0}(\mathbf{x})$ and $T^{1}(\mathbf{x})$ are bounded below by $\bar{T}_{1}$ and bounded above by $\bar{T}_{2}$ almost everywhere and if $\left|\bar{T}_{2}-\bar{T}_{1}\right|$ and $\|\overline{\mathbf{u}}\|_{1}$ are sufficiently small, then (2.1) has a unique solution in $\mathrm{Z}$.

Proof. Suppose $\left(\mathbf{u}_{i}, T_{i}\right) \in \mathbf{Z}, i=1,2$ are two solutions to (2.1). Then letting $\widetilde{\mathbf{u}}=\mathbf{u}_{1}-\mathbf{u}_{2}$ and $\widetilde{T}=T_{1}-T_{2}$, we have

$$
\begin{aligned}
& a_{0}(\tilde{\mathbf{u}}, \mathbf{v})+b_{0}\left(\mathbf{u}_{1}, \widetilde{\mathbf{u}}, \mathbf{v}\right)+b_{0}\left(\widetilde{\mathbf{u}}, \mathbf{u}_{2}, \mathbf{v}\right)+\widehat{\alpha}(\widetilde{T} \mathbf{g}, \mathbf{v})=0 \\
& a_{1}(\tilde{T}, \psi)+b_{1}\left(\mathbf{u}_{1}, \tilde{T}, \psi\right)+b_{1}\left(\widetilde{\mathbf{u}}, T_{2}, \psi\right)+\widehat{\kappa} h(\tilde{T}, \psi)_{\Gamma_{1}}=0
\end{aligned}
$$

for $(\mathbf{v}, \psi) \in \mathbf{V}$. Setting $\mathbf{v}=\widetilde{\mathbf{u}}$ and $\psi=\widetilde{T}$, we obtain, using (2.4) and (2.5), that

$$
a_{0}(\widetilde{\mathbf{u}}, \widetilde{\mathbf{u}}) \leq C_{3}\left\|\mathbf{u}_{2}\right\|_{1}|\widetilde{\mathbf{u}}|_{1}^{2}+\hat{\alpha}\|\tilde{T}\|_{0}\|\tilde{\mathbf{u}}\|_{0}
$$

and

$$
a_{1}(\tilde{T}, \tilde{T})+\widehat{\kappa} h\left\|\left.\tilde{T}\right|_{0, \Gamma_{1}} ^{2} \leq\right\| T_{2}-T_{0}\left\|_{L^{\infty}}\right\| \tilde{\mathbf{u}}\left\|_{0}\right\| \tilde{T} \|_{1},
$$

where $T_{0}=\frac{1}{2}\left(\bar{T}_{1}+\bar{T}_{2}\right)$. This implies

$$
\left(\widehat{\nu}-C_{3}\left\|\mathbf{u}_{2}\right\|_{1}\right)|\widetilde{\mathbf{u}}|_{1}^{2} \leq \widehat{\alpha} C_{7} C_{12}|\widetilde{\mathbf{u}}|_{1} \mid \tilde{T} \|_{1}
$$

and

$$
\widehat{\kappa}\left\|\left.\widetilde{T}\right|_{1} ^{2} \leq C_{7}\right\| T_{2}-T_{0}\left\|_{L^{\infty}}|\widetilde{\mathbf{u}}|_{1}\right\| \widetilde{T} \|_{1},
$$

where $\|\psi\|_{0} \leq C_{12}\|\psi\|_{1}$ for $\psi \in V_{1}$. Hence if

$$
\widehat{\kappa}\left(\widehat{\nu}-C_{3} \|\left.\mathbf{u}_{2}\right|_{1}\right)-\widehat{\alpha} C_{7}^{2} C_{12}\left\|T_{2}-T_{0}\right\|_{L^{\infty}}>0
$$

then $|\widetilde{\mathbf{u}}|_{1}=\|\tilde{T}\|_{1}=0$ and thus $\left(\mathbf{u}_{1}, T_{1}\right)=\left(\mathbf{u}_{2}, T_{2}\right)$.

From Theorem 2.2 and (2.7), we have

$$
|| T_{2}-\left.T_{0}\right|_{L^{\infty}} \leq \frac{\bar{T}_{2}-\bar{T}_{1}}{2}
$$

and

$$
\left\|\mathbf{u}_{2}\right\|_{1} \leq \frac{2 C_{7}}{\widehat{\nu}} \widehat{\alpha}\left\|T_{2}-T_{0}\right\|_{0}+\frac{2 C_{3}}{\widehat{\nu}}\left(\|\overline{\mathbf{u}}\|_{1}^{2}+\|\overline{\mathbf{u}}\|_{1}\right) .
$$

Thus, if $\left|\bar{T}_{2}-\bar{T}_{1}\right|$ and $\|\left.\overline{\mathbf{u}}\right|_{1}$ are sufficiently small then (2.1) has a unique solution in $\mathbf{Z}$. 
3. Existence of Optimal Controls and Necessary Optimality Conditions. In this section, we show the existence of optimal solutions for the minimization problem (1.1)(1.3) and estabilish a necessary optimality condition. Let us first assume that $\mathcal{C}$ is a closed convex subset of $L^{2}\left(\Gamma_{1}\right)$. For example $\mathcal{C}$ can be defined to be

$$
\mathcal{C}=\left\{g \in L^{2}\left(\Gamma_{1}\right): \bar{T}_{1} \leq g \leq \bar{T}_{2} \text { almost everywhere }\right\}
$$

or $\mathcal{C}=L^{2}\left(\Gamma_{1}\right)$. Let us denote the set

$$
\mathbf{S}(g)=\{(\mathbf{u}, T, g) \in \mathbf{X}=\mathbf{Z} \times \mathcal{C}: g \in \mathcal{C} \text { and }(\mathbf{u}, \mathrm{T}) \text { satisfies }(2.1)\} .
$$

Let us define the cost functional $\mathcal{J}(\mathbf{u}, T, g)$ to be

$$
\mathcal{J}(\mathbf{u}, T, g)=\boldsymbol{\varphi}(\mathbf{u}, T)+\frac{\delta}{2}\|g\|_{0, \Gamma_{1}}^{2},
$$

and cast the control problems in the following abstract setting: For $\mathbf{x}=(\mathbf{u}, T, g) \in \mathbf{X}=$ $\mathrm{Z} \times \mathcal{C}$ with

$$
\begin{gathered}
\text { Minimize } \mathcal{J}(\mathbf{x}) \\
\mathbf{x} \in \mathbf{X} \\
\text { subject to } \mathbf{E}(\mathbf{x})=\mathbf{0} \text { and } g \in \mathcal{C},
\end{gathered}
$$

where the equality constraint $\mathbf{E}: \mathbf{X} \rightarrow \mathbf{Y}=\mathbf{V}^{*}$ represents the state equations (2.1),

$$
\langle\mathbf{E}(\mathbf{x}),(\mathbf{v}, \psi)\rangle_{\mathbf{V}^{*} \times \mathbf{V}}=a(\mathbf{z},(\mathbf{v}, \psi))+b(\mathbf{u}, \mathbf{z},(\mathbf{v}, \psi))+\hat{\kappa} h(T-g, \psi)_{\Gamma_{1}}+(\hat{\alpha} T \mathbf{g}, \mathbf{v})
$$

for $(\mathbf{v}, \psi) \in \mathbf{V}$, where

$$
\begin{aligned}
& a(\mathbf{z},(\mathbf{v}, \psi))=a_{0}(\mathbf{u}, \mathbf{v})+a_{1}(T, \psi) \\
& b(\mathbf{u}, \mathbf{z},(\mathbf{v}, \psi))=b_{0}(\mathbf{u}, \mathbf{u}, \mathbf{v})+b_{1}(\mathbf{u}, T, \psi) .
\end{aligned}
$$

Then, we have the existence of solutions to the optimal control problem.

Theorem 3.1. Consider the minimization problem:

$$
\begin{gathered}
\text { Minimize } \mathcal{J}(\mathbf{u}, T, g) \\
(\mathbf{u}, T, g) \in \mathbf{S}(g) \times \mathcal{C}
\end{gathered}
$$

where $\mathcal{C}$ is a closed convex subset of $L^{2}\left(\Gamma_{1}\right)$. Assume that the function

$$
\boldsymbol{\varphi}(\mathbf{z}): \mathbf{z}=(\mathbf{u}, T) \in \mathbf{Z} \rightarrow \mathbb{R}^{+}
$$

is convex and lower semicontinuous and satisfies $\varphi(\mathbf{z}) \leq c_{1}\|\mathbf{z}\|_{1}^{2}+c_{2}$ for $c_{1}, c_{2} \in \mathbb{R}^{+}$. Then the minimization problem has a solution. 
Proof. Let $\left(\mathbf{u}_{k}, T_{k}, g_{k}\right) \in \mathbf{S}\left(g_{k}\right) \times \mathcal{C}$ be a minimizing sequence. Since $\delta>0,\left\|g_{k}\right\|_{0, \Gamma_{1}}$ is uniformly bounded in $k$ and thus from (2.9) so is $\left\|\left(\mathbf{u}_{k}, T_{k}\right)\right\|_{1}$. Hence there exists a subsequence of $\{k\}$, which will be denoted by the same index, such that $\left(\mathbf{u}_{k}, T_{k}, g_{k}\right)$ converges weakly to $(\mathbf{u}, T, g) \in \mathbf{Z} \times \mathcal{C}$, since $\mathbf{V} \times L^{2}\left(\Gamma_{1}\right)$ is a Hilbert space and $\mathcal{C}$ is a closed and convex set. Since $H^{1}(\Omega)$ is compactly embedded into $L^{4}(\Omega)$, it follows from Lemma 2.1 that

$$
b_{0}\left(\mathbf{u}_{k}, \mathbf{u}_{k}, \mathbf{v}\right) \rightarrow b_{0}(\mathbf{u}, \mathbf{u}, \mathbf{v}) \forall \mathbf{v} \in \mathbf{V}_{0}
$$

and

$$
b_{1}\left(\mathbf{u}_{k}, T_{k}, \psi\right) \rightarrow b_{0}(\mathbf{u}, T, \psi) \forall \psi \in V_{1}
$$

which implies $(\mathbf{u}, T) \in \mathbf{S}(g)$. Now, since $\boldsymbol{\varphi}$ is convex and lower semicontinuous it follows from [3] that $(\mathbf{u}, T, g)$ minimizes $(3.1)$.

Assume that $\mathbf{x}^{*}=\left(\mathbf{z}^{*}, g^{*}\right)=\left(\mathbf{u}^{*}, T^{*}, g^{*}\right)$ denotes an optimal pair of (3.1). Then we have the following theorem.

Theorem 3.2. Assume that $\mathrm{x}^{*}$ is a regular point in the sense that

$$
0 \in \operatorname{int}\left\{\mathbf{E}^{\prime}\left(\mathbf{x}^{*}\right)\left(\mathbf{v}, \psi, \eta-g^{*}\right):(\mathbf{v}, \psi) \in \mathbf{V} \text { and } \eta \in \mathcal{C}\right\} .
$$

Then there exists Lagrange multipliers $(\boldsymbol{\zeta}, \lambda) \in \mathbf{V}$ such that

$$
a((\boldsymbol{\zeta}, \lambda),(\mathbf{v}, \psi))+b\left(\mathbf{v}, \mathbf{z}^{*},(\boldsymbol{\zeta}, \lambda)\right)+b\left(\mathbf{u}^{*},(\mathbf{v}, \psi),(\boldsymbol{\zeta}, \lambda)\right)+\left\langle\boldsymbol{\varphi}^{\prime}\left(\mathbf{z}^{*}\right),(\mathbf{v}, \psi)\right\rangle=0
$$

for $(\mathbf{v}, \psi) \in \mathbf{V}$ and

$$
\left(\delta g^{*}-\widehat{\kappa} h \lambda, \eta-g^{*}\right)_{\Gamma_{1}} \geq 0 \quad \forall \eta \in \mathcal{C} .
$$

Proof. It follows from [14] that if (3.2) is satisfied, then there exists a Lagrange multipliers $(\boldsymbol{\zeta}, \lambda) \in \mathbf{V}$ such that

$$
\left\langle\boldsymbol{\varphi}^{\prime}\left(\mathbf{z}^{*}\right),(\mathbf{v}, \psi)\right\rangle+\delta\left(g^{*}, \eta-g^{*}\right)_{\Gamma_{1}}+\mathbf{E}^{\prime}\left(\mathbf{x}^{*}\right)\left((\mathbf{v}, \psi), \eta-g^{*}\right) \geq 0
$$

for all $(\mathbf{v}, \psi) \in \mathbf{V}$ and $\eta \in \mathcal{C}$, that is

$$
\begin{gathered}
\left\langle\boldsymbol{\varphi}^{\prime}\left(\mathbf{z}^{*}\right),(\mathbf{v}, \psi)\right\rangle+\delta\left(g^{*}, \eta-g^{*}\right)_{\Gamma_{1}}+a((\boldsymbol{\zeta}, \lambda),(\mathbf{v}, \psi))+b\left(\mathbf{v}, \mathbf{z}^{*},(\boldsymbol{\zeta}, \lambda)\right) \\
+b\left(\mathbf{u}^{*},(\mathbf{v}, \psi),(\boldsymbol{\zeta}, \lambda)\right)-\widehat{\kappa} h\left(\eta-g^{*}, \lambda\right)_{\Gamma_{1}} \geq 0
\end{gathered}
$$

for all $(\mathbf{v}, \psi) \in \mathbf{V}$ and $\eta \in \mathcal{C}$. Setting $(\mathbf{v}, \psi)=0$, we obtain (3.4). Next, setting $\eta=g^{*}$ in (3.5), we obtain (3.3).

Concerning the regular point condition (3.2), we have 
LEMMA 3.3. If $g^{*} \in \operatorname{int}(\mathcal{C})$ then the regular point condition (3.2) is equivalent to the following condition. Suppose $\mathbf{y}=(\mathbf{w}, \theta) \in \mathbf{V}$ satisfies

$$
\begin{aligned}
& a(\mathbf{y},(\mathbf{v}, \psi))+b\left(\mathbf{w}, \mathbf{z}^{*},(\mathbf{v}, \psi)\right)+b\left(\mathbf{u}^{*}, \mathbf{y},(\mathbf{v}, \psi)\right)=0 \quad \forall(\mathbf{v}, \psi) \in \mathbf{V} \\
& \text { and } \quad \theta=0 \quad \text { on } \quad \Gamma_{1} .
\end{aligned}
$$

Then $\mathbf{y}=\mathbf{0}$.

Proof. If $g^{*} \in \operatorname{int}(\mathcal{C})$ then $(3.2)$ is equivalent to $\mathbf{G}=\mathbf{E}^{\prime}\left(\mathbf{x}^{*}\right)$ is surjective. Define the linear map $\mathbf{F} \in \mathcal{L}\left(\mathbf{V} \times L^{2}\left(\Gamma_{1}\right), \mathbf{V}\right)$ by $\mathbf{F}((\mathbf{w}, \theta), \eta)=\boldsymbol{\xi}$ where $\boldsymbol{\xi} \in \mathbf{V}$ is a unique solution to

$$
a(\boldsymbol{\xi},(\mathbf{v}, \psi))+b\left(\mathbf{w}, \mathbf{z}^{*},(\mathbf{v}, \psi)\right)+b\left(\mathbf{u}^{*},(\mathbf{w}, \theta),(\mathbf{v}, \psi)\right)+\widehat{\kappa} h(\boldsymbol{\xi}-\eta, \psi)_{\Gamma_{1}}=0 \quad \forall(\mathbf{v}, \psi) \in \mathbf{V}
$$

Then, since $H^{1}(\Omega)$ is embedded compactly to $L^{4}(\Omega)$, by Lemma $2.1, \mathbf{F}$ is compact. Thus, it follows from Banach closed range and Riesz-Schauder theorems that $\mathbf{E}^{\prime}\left(\mathbf{x}^{*}\right)((\mathbf{w}, \theta), \eta)$ is surjective if and only if $\operatorname{ker}\left(\mathbf{G}^{*}\right)=\{0\}$, which is equivalent to (3.6).

Finally, if $\mathcal{C}=L^{2}\left(\Gamma_{1}\right)$ and the cost functional is given as in (1.1) then (3.3)-(3.4) can be equivalently written as

$$
\left\{\begin{array}{l}
a_{0}(\boldsymbol{\zeta}, \mathbf{v})+b_{0}(\mathbf{u}, \mathbf{v}, \boldsymbol{\zeta})+b_{0}(\mathbf{v}, \mathbf{u}, \boldsymbol{\zeta})+b_{1}(\mathbf{v}, T, \lambda)+(\nabla \times \mathbf{u}, \nabla \times \mathbf{v})=0 \\
a_{1}(\lambda, \nabla \psi)+b_{1}(\mathbf{u}, \psi, \lambda)+\widehat{\alpha}(\boldsymbol{\zeta}, \psi \mathbf{g})+\widehat{\kappa} h(\lambda, \psi)_{\Gamma_{1}}=0 \\
\delta g-\widehat{\kappa} h \lambda=0 \quad \text { on } \Gamma_{1}
\end{array}\right.
$$

for all $(\mathbf{v}, \psi) \in \mathbf{V}$.

To facilitate the computational discussion, let us collect the necessary conditions of optimality (2.1) and (3.7) and recast them by using the vector decomposition of $\mathrm{L}^{2}(\Omega)$, (see $[10]), \mathbf{L}^{2}(\Omega)=\mathbf{H}_{0}+\left\{\nabla \phi: \phi \in H^{1}(\Omega)\right\}$, and by introducing pressure $p$ and adjoint pressure $\pi$. We obtain: For $\mathbf{u} \in \mathbf{V}_{0}+\overline{\mathbf{u}}, T \in V_{1}+\bar{T}, \boldsymbol{\zeta} \in \mathbf{H}_{0}^{1}(\Omega), \lambda \in V_{1}, p \in L_{0}^{2}(\Omega)$ and $\pi \in L_{0}^{2}(\Omega)$,

$$
\left\{\begin{array}{l}
a_{0}(\mathbf{u}, \mathbf{v})+b_{0}(\mathbf{u}, \mathbf{u}, \mathbf{v})+c(\mathbf{v}, p)+\widehat{\alpha}(T \mathbf{g}, \mathbf{v})=0 \forall \mathbf{v} \in \mathbf{H}_{0}^{1}(\Omega), \\
c(\mathbf{u}, q)=0 \forall q \in L_{0}^{2}(\Omega), \\
a_{1}(T, \psi)+b_{1}(\mathbf{u}, T, \psi)+\widehat{\kappa} h(T-g, \psi)_{\Gamma_{1}}=0 \forall \psi \in V_{1}
\end{array}\right.
$$




$$
\left\{\begin{array}{r}
a_{0}(\boldsymbol{\zeta}, \mathbf{v})+b_{0}(\mathbf{u}, \mathbf{v}, \zeta)+b_{0}(\mathbf{v}, \mathbf{u}, \zeta)+b_{1}(\mathbf{v}, T, \lambda)+c(\mathbf{v}, \pi) \\
+(\nabla \times \mathbf{u}, \nabla \times \mathbf{v})=0 \quad \forall \mathbf{v} \in \mathbf{H}_{0}^{1}(\Omega) \\
c(\boldsymbol{\zeta}, q)=0 \quad \forall q \in L_{0}^{2}(\Omega), \\
a_{1}(\lambda, \nabla \psi)+b_{1}(\mathbf{u}, \psi, \lambda)+\widehat{\alpha}(\boldsymbol{\zeta}, \psi \mathbf{g})+\widehat{\kappa} h(\lambda, \psi)_{\Gamma_{1}}=0 \quad \forall \psi \in V_{1} \\
\delta g-\widehat{\kappa} h \lambda=0 \quad \text { on } \Gamma_{1} .
\end{array}\right.
$$

The system (3.8)-(3.9) forms the necessary conditions of optimality that optimal states and control must satisfy. This system will also be called the optimality system.

4. Computational Methods. In this section we describe a computational method to find the optimal control by solving the optimality system (3.8)-(3.9).

4.1. Finite Element Approximations. A finite element discretization of the optimality system (3.8)-(3.9) is defined in the usual manner. First one chooses families of finite dimensional subspaces $X_{h} \subset H^{1}(\Omega)$ and $S_{h} \subset L^{2}(\Omega)$. We let $X_{h}^{0}=X_{h} \cap H_{0}^{1}(\Omega)$, $S_{h}^{0}=S_{h} \cap L_{0}^{2}(\Omega)$ and $\mathbf{X}_{h}=\left[X_{h}\right]^{2}$. These families are parameterized by a parameter $h$ that tends to zero; commonly, $h$ is chosen to be some measure of the grid size. These finite dimensional function spaces are defined on an approximate domain $\Omega_{h}$. For simplicity we will state our results in this section by assuming $\Omega_{h}=\Omega$. We assume that these finite element spaces satisfy the following approximation properties (see, [7, 10]): there exist an integer $k$ and a constant $C$, independent of $h, \mathbf{v}, q$ and $\psi$, such that

$$
\begin{aligned}
& \inf _{\mathbf{v}^{h} \in \mathbf{V}^{h}}\left\|\mathbf{v}-\mathbf{v}^{h}\right\|_{1} \leq C h^{m}\|\mathbf{v}\|_{m+1} \quad \forall \mathbf{v} \in \mathbf{H}^{m+1}(\Omega), 1 \leq m \leq k, \\
& \inf _{q^{h} \in S^{h}}\left\|q-q^{h}\right\|_{0} \leq C h^{m}\|q\|_{m} \quad \forall q \in H^{m}(\Omega) \cap L_{0}^{2}(\Omega), 1 \leq m \leq k,
\end{aligned}
$$

and

$$
\inf _{\psi^{h} \in V^{h}}\left\|\psi-\psi^{h}\right\|_{1} \leq C h^{m}\|\psi\|_{m+1} \quad \forall \psi \in H^{m+1}(\Omega), 1 \leq m \leq k .
$$

Here we may choose any pair of subspaces $X_{h}$ and $S_{h}$ such that $\mathbf{X}_{h}^{0}$ and $S_{h}^{0}$ can be used for finding finite element approximations of solutions of the Navier-Stokes equations with homogeneous Dirichlet conditions. Thus, we make the following standard assumptions, which are exactly those employed in well-known finite element methods for the Navier-Stokes equations. Next, we assume the inf-sup condition: there exists a constant $C$, independent of $h$, such that

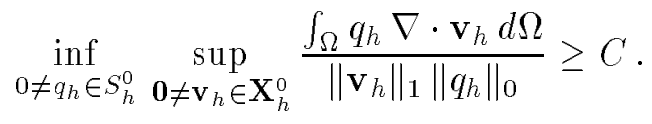


This condition assures the stability of finite element discretizations of the Navier-Stokes equations and also that of the optimlity system (3.8)-(3.9). The references [9] and [6] may also be consulted for a catalogue of finite element subspaces that meet the requirements of the above approximation properties and the inf-sup condition. We also define $Z_{h}$ to be $Z_{h} \subset L^{2}\left(\Gamma_{1}\right)$.

Once the approximating subspaces have been chosen, we look for an approximate optimal solution $\left(\mathbf{u}_{h}, p_{h}, T_{h}, \boldsymbol{\zeta}_{h}, \pi_{h}, \lambda_{h}, g_{h}\right) \in \mathbf{X}_{h} \times S_{h}^{0} \times X_{h} \times \mathbf{X}_{h}^{0} \times S_{h}^{0} \times X_{h} \times Z_{h}$ by solving the discrete optimality system of equations

$$
\left\{\begin{array}{rr}
\left(\delta g_{h}-\widehat{\kappa} h \lambda_{h}, z_{h}\right)_{\Gamma_{1}}=0 \quad \forall z_{h} \in Z_{h} \cap L^{2}\left(\Gamma_{1}\right), \\
a_{0}\left(\mathbf{u}_{h}, \mathbf{v}_{h}\right)+b_{0}\left(\mathbf{u}_{h}, \mathbf{u}_{h}, \mathbf{v}_{h}\right)+c\left(\mathbf{v}_{h}, p_{h}\right)+\widehat{\alpha}\left(T_{h} \mathbf{g}, \mathbf{v}_{h}\right)=0 & \forall \mathbf{v}_{h} \in \mathbf{X}_{h}^{0}, \\
a_{1}\left(T_{h}, \psi_{h}\right)+b_{1}\left(\mathbf{u}, T_{h}, \psi_{h}\right)+\widehat{\kappa} h\left(T_{h}-g, \psi\right)_{\Gamma_{1}}=0 & \forall \psi_{h} \in X_{h} \cap V_{1}, \\
c\left(\mathbf{u}_{h}, q_{h}\right)=0 & \forall q_{h} \in S_{h}^{0}, \\
a_{0}\left(\boldsymbol{\zeta}_{h}, \mathbf{v}_{h}\right)+b_{0}\left(\mathbf{u}_{h}, \mathbf{v}_{h}, \boldsymbol{\zeta}_{h}\right)+b_{0}\left(\mathbf{v}_{h}, \mathbf{u}_{h}, \boldsymbol{\zeta}_{h}\right)+c\left(\mathbf{v}_{h}, \pi_{h}\right)+b_{1}\left(\mathbf{v}_{h}, T_{h}, \lambda_{h}\right) \\
+\left(\nabla \times \mathbf{u}_{h}, \nabla \times \mathbf{v}_{h}\right)=0 \quad \forall \mathbf{v}_{h} \in \mathbf{X}_{h}^{0}, \\
a_{1}\left(\lambda_{h}, \psi_{h}\right)+b_{1}\left(\mathbf{u}_{h}, \psi_{h}, \lambda_{h}\right)+\hat{\alpha}\left(\psi_{h}, \mathbf{g} \boldsymbol{\zeta}_{h}\right)+\widehat{\kappa} h\left(\psi_{h}, \lambda_{h}\right)_{\Gamma_{1}}=0 \\
\forall \psi_{h} \in X_{h} \cap V_{1}(\Omega), \\
c\left(\zeta_{h}, q_{h}\right)=0 & \forall q_{h} \in S_{h}^{0} .
\end{array}\right.
$$

We next briefly sketch the proof of optimal error estimates. We first prove optimal error estimates for the approximations of the linearized optimality system. Then by a careful choice of spaces and operators we can fit the optimality system into the framework of BrezziRappaz-Raviart theory (see [10]). By verifying all the requirements of that theory, we obtain optimal error estimates for the approximation of the optimality system of equations.

Theorem 4.1. Assume $(\mathbf{u}, T, p, \zeta, \lambda, \pi) \in \mathbf{H}^{m+1}(\Omega) \times H^{m}(\Omega) \times H^{m+1}(\Omega) \times \mathbf{H}^{m+1}(\Omega) \times$ $H^{m}(\Omega) \times H^{m+1}(\Omega)$ is a nonsingular solution of the optimality system (3.8)-(3.9). Then for each sufficiently small $h$, the approximate optimality system (3.10) has a unique solution $\left(\mathbf{u}^{h}, T^{h}, p^{h}, \boldsymbol{\zeta}^{h}, \lambda^{h}, \pi^{h}\right) \in \mathbf{X}_{h} \times S_{h}^{0} \times X_{h} \times \mathbf{X}_{h}^{0} \times S_{h}^{0} \times X_{h}$ in a neighborhood of $(\mathbf{u}, T, p, \boldsymbol{\zeta}, \boldsymbol{\lambda}, \pi)$, such that

$$
\begin{aligned}
& \left\|\mathbf{u}-\mathbf{u}^{h}\right\|_{1}+\left\|p-p^{h}\right\|_{0}+\left\|T-T^{h}\right\|_{1}+\left\|\boldsymbol{\zeta}-\boldsymbol{\zeta}^{h}\right\|_{1}+\left\|\pi-\pi^{h}\right\|_{0}+\left\|\lambda-\lambda^{h}\right\|_{1} \\
& \leq C h^{m}\left\{\|\mathbf{u}\|_{m+1}+\|p\|_{m}+\|T\|_{m+1}+\|\boldsymbol{\zeta}\|_{m+1}+\|\pi\|_{m}+\|\lambda\|_{m+1}\right\} .
\end{aligned}
$$

We employ Newton's iteration method to solve this finite dimensional nonlinear system of equations. 
4.2. Newtons Method. The Newton's method based on exact Jacobian for solving the discrete optimality system is given as follows:

$1^{\circ}$ Triangulate the flow domain with a sufficiently small mesh size $h$; choose finite element spaces $\mathbf{X}_{h}$ and $S_{h}$; choose an initial guess $\left(\mathbf{u}^{0}, T^{0}, p^{0}, \boldsymbol{\zeta}^{0}, \lambda^{0}, \pi^{0}, g^{0}\right)$;

$2^{\circ}$ For $n=1,2, \cdots$, compute $\left(\mathbf{u}^{n}, T^{n}, p^{n}, \boldsymbol{\zeta}^{n}, \lambda^{n}, \pi^{n}, g^{n}\right)$ from the following discrete system of equations:

$$
\left\{\begin{array}{r}
\left(\delta g^{n}-\widehat{\kappa} h \lambda^{n}, z_{h}\right)_{\Gamma_{1}}=0 \quad \forall z_{h} \in Z_{h} \cap L^{2}\left(\Gamma_{1}\right), \\
a_{0}\left(\mathbf{u}^{n}, \mathbf{v}_{h}\right)+b_{0}\left(\mathbf{u}^{n-1}, \mathbf{u}^{n}, \mathbf{v}_{h}\right)+b_{0}\left(\mathbf{u}^{n}-\mathbf{u}^{n-1}, \mathbf{u}^{n-1}, \mathbf{v}_{h}\right)+c\left(\mathbf{v}_{h}, p^{n}\right) \\
+\widehat{\alpha}\left(T^{n} \mathbf{g}, \mathbf{v}_{h}\right)=0 \quad \forall \mathbf{v}_{h} \in \mathbf{X}_{h}^{0}, \\
c\left(\mathbf{u}^{n}, q_{h}\right)=0 \quad \forall q_{h} \in S_{h}^{0}, \\
a_{1}\left(T^{n}, \psi_{h}\right)+b_{1}\left(\mathbf{u}^{n}, \psi_{h}, T^{n-1}\right)+b_{1}\left(\mathbf{u}^{n-1}, \psi_{h}, T^{n}-T^{n-1}\right) \\
+\widehat{\kappa} h\left(T^{n}-g^{n}, \psi_{h}\right)_{\Gamma_{1}}=0 \quad \forall \psi_{h} \in X_{h} \cap V_{1}, \\
a_{0}\left(\boldsymbol{\zeta}^{n}, \mathbf{v}_{h}\right)+b_{0}\left(\mathbf{u}^{n}-\mathbf{u}^{n-1}, \mathbf{v}_{h}, \boldsymbol{\zeta}^{n-1}\right)+c\left(\mathbf{v}_{h}, \pi^{n}\right)+b_{0}\left(\mathbf{v}_{h}, \mathbf{u}^{n-1}, \boldsymbol{\zeta}^{n}-\boldsymbol{\zeta}^{n-1}\right) \\
+b_{0}\left(\mathbf{u}^{n-1}, \mathbf{v}_{h}, \boldsymbol{\zeta}^{n}\right)+b_{0}\left(\mathbf{v}_{h}, \mathbf{u}^{n}, \boldsymbol{\zeta}^{n-1}\right)+c\left(\mathbf{v}_{h}, \pi^{n}\right) \\
+\left(\nabla \times \mathbf{u}^{n}, \nabla \times \mathbf{v}_{h}\right)=0 \forall \mathbf{v}_{h} \in \mathbf{X}_{h}^{0}, \\
c\left(\boldsymbol{\zeta}^{n}, q_{h}\right)=0 \quad \forall q_{h} \in S_{h}^{0}, \\
a_{1}\left(\lambda^{n}, \psi_{h}\right)+b_{1}\left(\mathbf{u}^{n}, \psi_{h}, \lambda^{n-1}\right)+b_{1}\left(\mathbf{u}^{n-1}, \psi_{h}, \lambda^{n}-\lambda^{n-1}\right)+\widehat{\alpha}\left(\psi_{h}, \boldsymbol{\zeta}^{n}\right) \\
+\widehat{\kappa} h\left(\psi_{h}, \lambda^{n}\right)_{\Gamma_{1}}=0 \quad \forall \psi_{h} \in X_{h} \cap V_{1} .
\end{array}\right.
$$

At each Newton's iteration, we solve the linear system of equations by Gaussian eliminations for banded matrices. Under suitable assumptions, Newton's method converges at a quadratic rate to the finite element solution $\left(\mathbf{u}_{h}, T_{h}, p_{h}, \boldsymbol{\zeta}_{h}, \lambda_{h}, \pi_{h}, g_{h}\right)$. Quadratic convergence of Newton's method is valid within a contraction ball. In practice we normally first perform a few successive approximations and then switch to the Newton's method. The successive approximations are defined by replacing the second, fourth, fifth and sixth equations in the Newton's iterations by

$$
\left\{\begin{array}{r}
a_{0}\left(\mathbf{u}^{n}, \mathbf{v}_{h}\right)+b_{0}\left(\mathbf{u}^{n-1}, \mathbf{u}^{n}, \mathbf{v}_{h}\right)+c\left(\mathbf{v}_{h}, p^{n}\right)+\widehat{\alpha}\left(T^{n} \mathbf{g}, \mathbf{v}_{h}\right)=0, \\
a_{1}\left(T^{n}, \psi_{h}\right)+b_{1}\left(\mathbf{u}^{n-1}, \psi_{h}, T^{n}\right)+\widehat{\kappa} h\left(T^{n}-g^{n}, \psi_{h}\right)_{\Gamma_{1}}=0, \\
a_{0}\left(\boldsymbol{\zeta}^{n}, \mathbf{v}_{h}\right)+b_{0}\left(\mathbf{u}^{n-1}, \mathbf{v}_{h}, \boldsymbol{\zeta}^{n}\right)+b_{0}\left(\mathbf{v}_{h}, \mathbf{u}^{n}, \boldsymbol{\zeta}^{n-1}\right)+c\left(\mathbf{v}_{h}, \pi^{n}\right) \\
+\left(\nabla \times \mathbf{u}^{n}, \nabla \times \mathbf{v}_{h}\right)=0 \\
a_{1}\left(\lambda^{n}, \psi_{h}\right)+b_{1}\left(\mathbf{u}^{n-1}, \psi_{h}, \lambda^{n}\right)+\widehat{\alpha}\left(\psi_{h}, \boldsymbol{\zeta}^{n}\right)+\widehat{\kappa} h\left(\psi_{h}, \lambda^{n}\right)_{\Gamma_{1}}=0 .
\end{array}\right.
$$

In the case of the uncontrolled Navier-Stokes equations, the solution is unique for small Reynolds numbers and the successive approximations converge globally and linearly; see 
[7]. However, in the present case of an optimal system of equations for the Navier-Stokes equations, the solution is not shown to be unique and the successive approximation is not shown to be globally convergent, even for small Reynolds numbers. Our numerical experience seems to suggest that the global convergence of the successive approximations for the optimality system is still valid for small Reynolds numbers. Thus the combined successive approximations-Newton iterations gives an effective method for solving the discrete optimality system of equations.

5. Computational Results. We will consider two test examples for vorticity minimization using boundary temperature control. Both examples are related to optimization and control of vapuor transport process for crystal growth. Some related works are reported in [19], [13] and [5]. In [19], tracking temperature field in an ampoule using boundary temperature control is considered, tracking a desired history of the freezing interface location/motion in conduction driven solidification process using temperature control is considered [13] and some optimal control problems in combustion are discussed in [5].

5.1. Numerical Example 1. In this example, we consider the control of vorticity in a backward-facing-step channel flow. The vorticity is caused by the injection of flow at the inlet of the channel and we try to control the vorticity or the recirculation rather by adjusting the temperature at the top and bottom walls. A schematic of the backwardfacing-step channel is shown in Figure 1. The height of the step is 0.5 and that of the outflow boundary is 1 . The length of the very bottom of the channel is 5 and the total horizontal length is 6 . Figure 3 demonstrate the flow situation for high Reynolds numbers which is computed with $g=0$ (no control) and the following boundary conditions for velocity and temperature.

$$
\begin{aligned}
& \Gamma_{\text {in }}: \quad \mathbf{u}=(8(0.5-y)(1-y), 0) \quad T=0 \\
& \Gamma_{\text {out }}: \quad \mathbf{u}=((1-y) y, 0) \quad \frac{\partial T}{\partial n}=0 \\
& \Gamma_{\text {top }}: \quad \mathbf{u}=(0,0) \quad \frac{\partial T}{\partial n}=-h T \\
& \Gamma_{\text {bottom }}: \quad \mathbf{u}=(0,0) \quad \frac{\partial T}{\partial n}=-h T \\
& \Gamma_{s}: \quad \mathbf{u}=(0,0) \quad T=1 .
\end{aligned}
$$

The parameters were taken as follows: $\operatorname{Re}=200, \operatorname{Pr}=.72, G r=40,000$ and $h=1$. The computational domain is divided into around 350 triangles with refined grid near the corner, see Figure 2. The finite element spaces $\mathbf{X}_{h}$ and $X_{h}$ are chosen to be piecewise quadratic elements (for $\mathbf{u}_{h}$ and $T_{h}$ ) defined over triangles and the space $S_{h}$ is chosen to be piecewise linear element (for $p_{h}$ ) defined over the same triangles.

A recirculation appears at the corner region whose size increases with increasing Reynolds number. The objective is to shape the recirculation region by applying temperature control on the very top boundary $\Gamma_{\text {top }}$ and bottom boundary $\Gamma_{\text {bottom }}$.

We take the corner region of the channel $\Omega^{*}=(1,3) \times(0, .5)$, see Figure 1 , for vorticity minimization. The control is computed by solving the optimality system (3.9) $-(3.10)$ by applying finite element and Newtons method described in $\$ 4.1-2$. The parameter in the functional was chosen as $\delta=0.01$ and the adjoint state variables $\zeta, \pi$ and $\lambda$ were discretized 
using the same way as their state counterparts. At each Newton iteration a banded Gaussian elimination was used to solve the resulting linear system. We obtain the optimal solution typically in 7 Newton iterations.

Figure 4 gives the controlled velocity field $\mathbf{u}_{h}$. Figures 5 and 6 are the blow-up of the uncontrolled and controlled flows, respectively, at the corner of the backward-facing-step. Figures 7 and 8 are the control distributions on the top and bottom boundaries.

The values of the integral $\int_{\Omega^{*}}|\nabla \times \mathbf{u}|^{2} d \Omega$ without and with controls were .94 and .51 , respectively. We see that we achieved a reduction of $45.74 \%$ in the $L^{2}(\Omega)$-norm of the vorticity.

5.2. Numerical Example 2. This example is motivated by the transport process in high pressure chemical vapour deposition (CVD) reactors (see [10-11] and [6]). A typical vertical reactor, shown in Figure 9, is a classical configuration for the growth of compound semiconductors by metalorganic vapor phase epitaxy. The reactant gases are introduced at the top of the reactor and flow down to the substrate $\left(\Gamma_{2}\right)$ which is kept at high temperature. This means that least dense gas is closest to the substrate and the flow is likely to be affected by buoyancy driven convection. In order to have uniform growth rates and better compositional variations, it is essential to have flow field without recirculations.

Our objective here is to minimize the vorticity by adjusting the temperature at the side walls $\left(\Gamma_{1}\right)$ in order to obtain a flow field without recirculations and thereby obtain better vertical transport.

The geometry of the prototype reactor, depicted in Figure 9, has two outlet portions, $\Gamma_{o}$, and an inlet, $\Gamma_{i}$, whose widths are $1 / 3$. The size of the susceptor region $\Gamma_{2}$ and that of the side walls $\Gamma_{1}$ are 1 ; the height of the inlet port $\Gamma_{s}$ is $1 / 3$.

The boundary conditions for computations were as follows:

$$
\begin{array}{lll}
\Gamma_{i}: & \mathbf{u}=\left(0,-4\left(x-\frac{1}{3}\right)\left(\frac{2}{3}-x\right)\right) & T=0 \\
\Gamma_{0}: & \frac{\partial u}{\partial n}=\frac{\partial v}{\partial n}=0 & \frac{\partial T}{\partial n}=0 \\
\Gamma_{2}: & \mathbf{u}=(0,0) & T=1 \\
\Gamma_{1}: & \mathbf{u}=(0,0) & \frac{\partial T}{\partial n}=h(g-T) \\
\Gamma_{s}: & \mathbf{u}=(0,0) & T=0
\end{array}
$$

For the uncontrolled flow computations, we take $g=0$ and throughout the computations in this problem we take the Reynolds number to be $R e=100$, the Prandtl number to be $\operatorname{Pr}=.72$ and $h=1$. For the discretization, the finite element spaces were chosen to be the same as in the previous example.

We performed simulations with several values of $G r / R e^{2}$ for the uncontrolled case. The flow situations are shown in Figure 10a)-15a) and the corresponding vorticity in $L^{2}$ norm is given in Table-I. Two standing circulation appear near the susceptor due to natural convection which did not appear at all when $G r / R e^{2} \ll 1$. For the control simulations heating/cooling control was applied to the side walls $\Gamma_{1}$ with fixed inflow rate and vorticity cost was minimized with the parameter $\delta=0.01$. This control problem was solved using our optimal control techniques. 
The resulting flow fields for various $G r / R e^{2}$ values are shown in Figure 10b)-15b) and the corresponding vorticity in $L^{2}$ norm is given in Table-I. The control values on the side walls are given in Figure 16a)-b). We see, in Figure 10b)-15b), significant reduction in recirculation for the controlled flow. Our computational experiments (not reported here) indicate that for $G r / R \epsilon^{2} \gg 1$, thermal control mechanism on the side walls with fixed flow rates may be less effective for the elimination of recirculation.

\begin{tabular}{|l||c|c|c|c|c|c|}
\hline \hline$G r / R e^{2}$ & 0.9 & 1.0 & 1.1 & 1.2 & 1.3 & 1.4 \\
\hline Uncontrolled Vorticity & 0.1983 & 0.2600 & 0.3505 & 0.4712 & 0.6186 & 0.7878 \\
\hline Controlled Vorticity & 0.1126 & 0.1174 & 0.1113 & 0.1123 & 0.1147 & 0.1183 \\
\hline \hline
\end{tabular}

Table I. Uncontrolled and Controlled Vorticity in $L^{2}$ norm for different $G r / R e^{2}$

6. Conclusion. In this article we studied vorticity minimization problem in fluid flows using boundary temperature controls. We formulated the problem as constrained minimization problem with cost functional being the vorticity in the flow. We proved the existence of optimal solution and the existence of Lagrange multipliers. The necessary conditions of optimality was given characterizing the controls and optimal states. Newton's method combined with mixed finite element method is used to solve the necessary conditions of optimality. We finally solved two canonical problems demonstrating the feasibility of the approach. 


\section{REFERENCES}

[1] F. Abergal and Temam, On Some Control Problems in Fluid Mechanics, Theoretical and Computational Fluid Dynamics, 1 (1990), pp 303-325.

[2] M. Desai and K. Ito, Optimal Control of Navier-Stokes Equations, SIAM Journal on Optimization and Control, 32 (1994), pp 1428-1446.

[3] I. Ekeland and R. Temam, Analysis of Convex Variational Problems, Dunod, Paris, 1976.

[4] H.O. Fattorini and S. S. Sritharan, Existence of Optimal Controls for Viscous Flow Problems, Proceeding of the Royal Society of London, Series A, 439 (1992), pp 81-102.

[5] ___ Optimal Control Problems with State Constraints in Fluid Mechanics and Combustion, Preprint.

[6] D.I. Fotiadis, S. Keida and K.F. Jensen, Transport Phenomena in Vertical Reactors for Metalorganic Vapor Phase Epitaxy, Journal of Crystal Growth, 102 (1990), pp. 441-470.

[7] M.D.Gunzburger, Finite Element Methods for Viscous Incompressible Flows, Academic Press, Inc. London, 1989

[8] M. Gunzburger, L. Hou And T. Svobodney, Analysis and Finite Element Approximation of Optimal Control Problems for the Stationary Navier-Stokes Equations with Dirichlet Controls, Modél. Math. Anal. Numér., 25 (1991), pp. 711-748.

[9] ___ Analysis and Finite Element Approximation of Optimal Control Problems for the Stationary Navier-Stokes Equations with Distributed and Neumann Controls, Math. Comp., 57 (1991), pp. 123-151.

[10] V.Girault and P.A. Raviart, Finite Element Methods for Navier-Stokes Equations, Springer-Verlag, Berlin, 1984.

[11] K.Ito and S. S. Ravindran, Optimal Control of Thermally coupled Navier-Stokes equations, Proceedings of the 34th Conference on Decision and Control, IEEE, New Orleans, LA, (1995), pp 461-466.

[12] K.Ito, J. Scroggs and H.T. Tran, Mathematical Issues in Optimal Design of a Vapour Transport Reactors, In M.D. Gunzburger, editor, Flow Control, Springer Verlag, New York, 1995.

[13] S. Kang AND N. ZaBaRas, Control of the Freezing Interface Motion in Two-dimensional Solidification Process Using the Adjoint Method, International Journal for Numerical Methods in Engineering, 38, (1995), pp.63-80.

[14] H. Maurer and J. Zowe, First and Second Order Necessary and Sufficient Optimality Conditions for Infinite-dimensional Programming Problems, Math Programming, 16 (1979), pp. 98-110.

[15] S.S. Ravindran, Numerical Solutions of Optimal Boundary Control Problems for Navier-Stokes Flows, AIAA Paper, AIAA-96-4048, 6th AIAA/NASA/ISSMO Symposium on Multidisciplinary Analysis and Optimization, Sep. 5th 1996.

[16] S.S. SRitharan, An Optimal Control Problem in Exterior Hydrodynamics, In E.B. Lee, G. Chen, L. Markus and W. Littman, editors, New Trends and Applications of Distributed Parameter Systems, Marcel Dekker, New York, 1990.

[17] _ Dynamic programming of the Navier-Stokes equations, System and Control Letters, 16 (1991), pp. 299-307.

[18] —_ Optimal Feedback Control of Hydrodynamics: A Progress Report, In M.D. Gunzburger, editor, Flow Control, Springer Verlag, New York, 1995.

[19] D.A. Tortorrelli and M.M. Tiller and J.A. Dantzig, Optimal Design of Nonlinear Parabolic Systems. Part I: Fixed Spatial Domain with Applications to Process Optimization, Comput. Methods Appl. Mech. Engrg., 113, (1994), pp. 141-155.

[20] G.M. Troinniello, Elliptic Differential Equations and Obstacle Problems, Plenum Press, New York, 1987. 


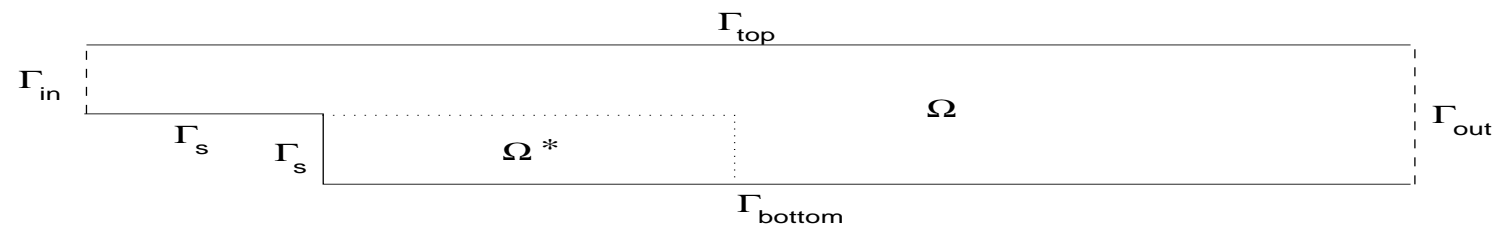

FIG. 1. Schematic of backward-facing-step channel.

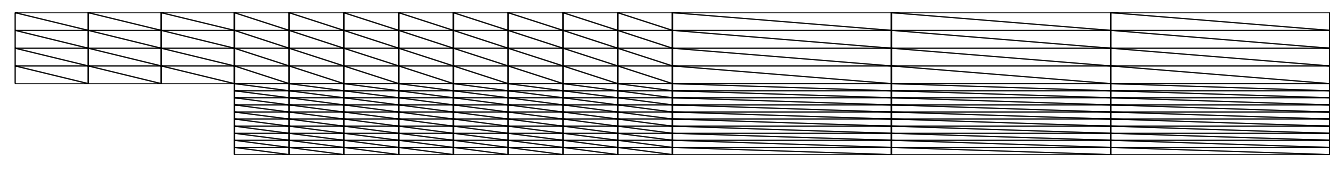

FIG. 2. Triangulation of the channel. 


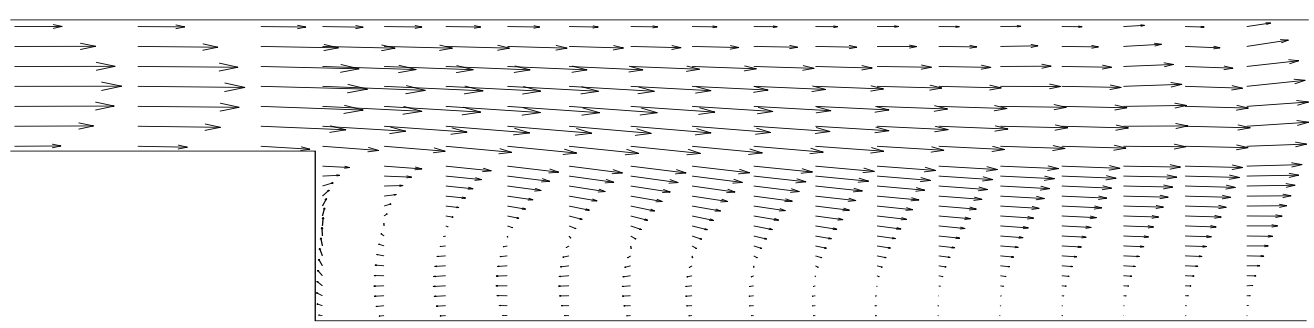

FIG. 3. Uncontrolled Channel Flow at Re=200

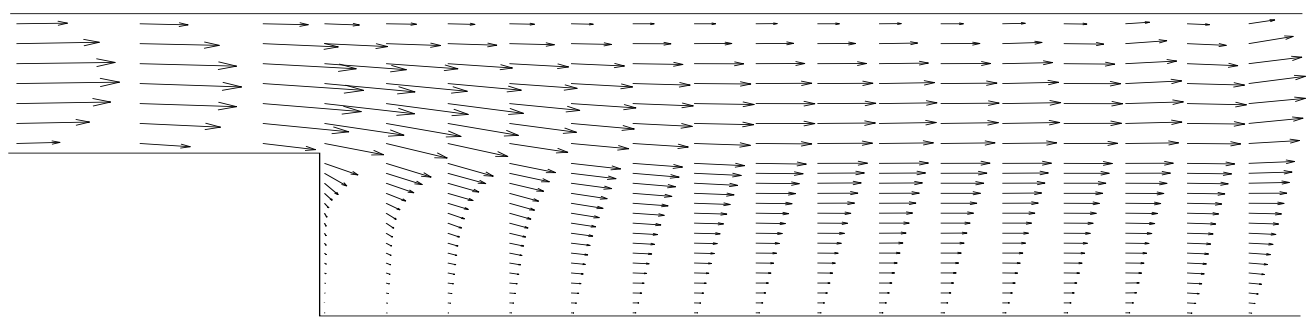

FIG. 4. Controlled Channel Flow at $\mathrm{Re}=200$ 


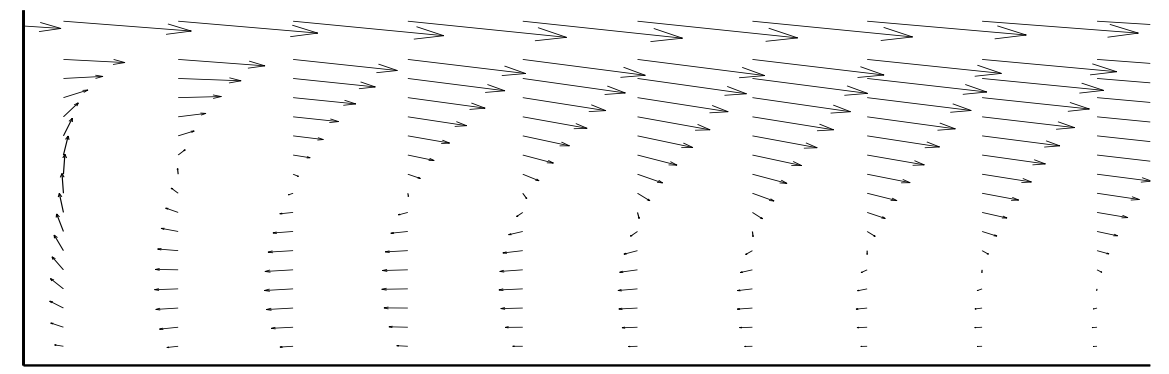

FIG. 5. Partial enlargement of FIG. 3

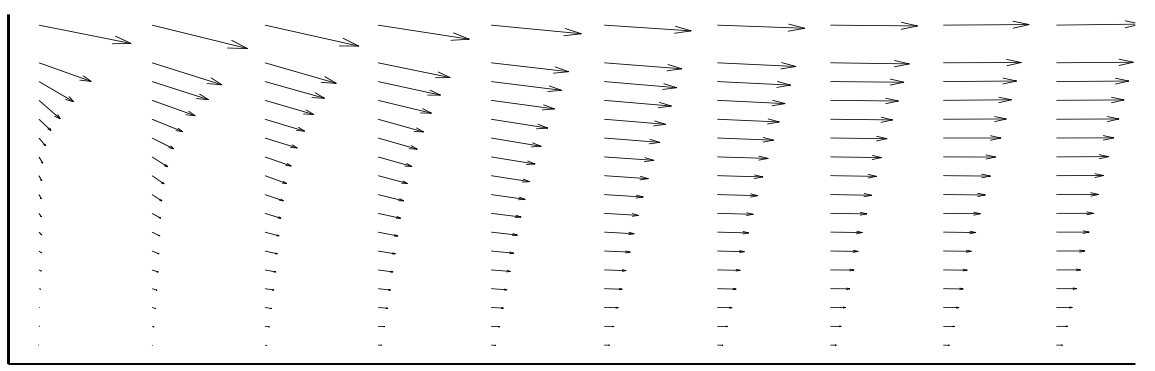

FIG. 6. Partial enlargement of FIG. 4 


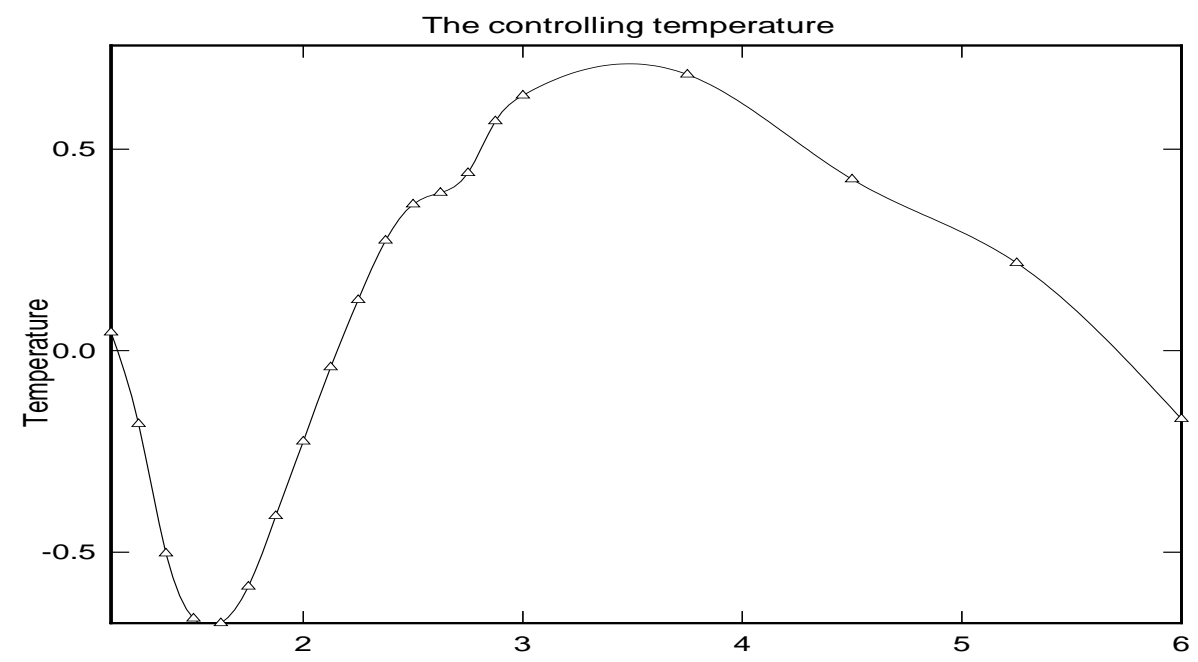

FIG. 7. Control on the boundary $\Gamma_{\text {bottom }}$

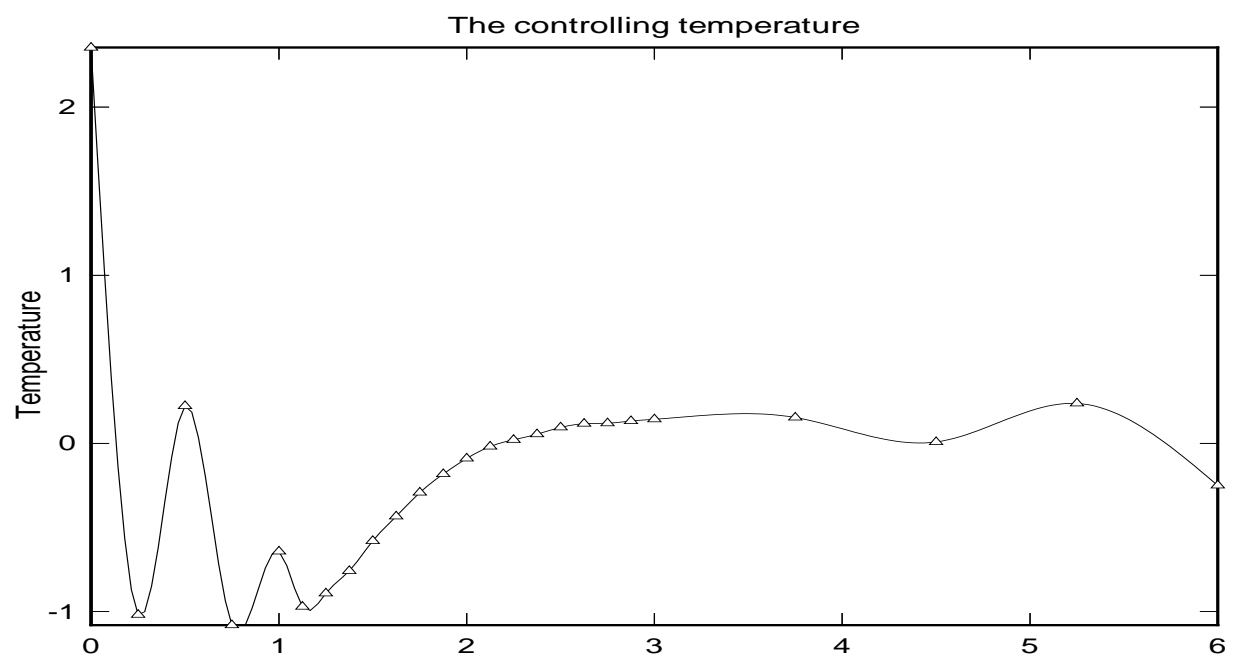

FIG. 8. Control on the boundary $\Gamma_{\text {top }}$ 


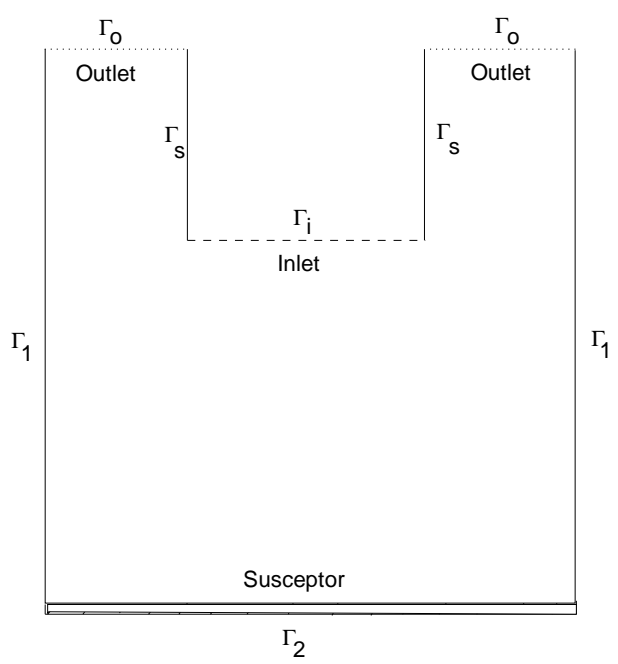

FIG. 9. Schematic of the flow domain

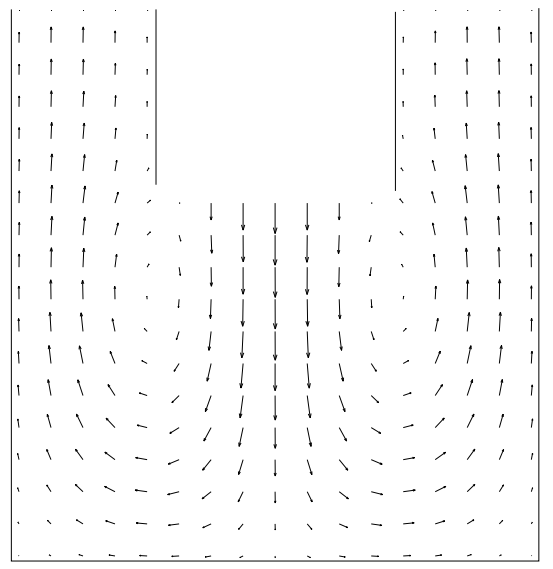

FIG. 10a) Uncontrolled flow for $G r / R e^{2}=.9$

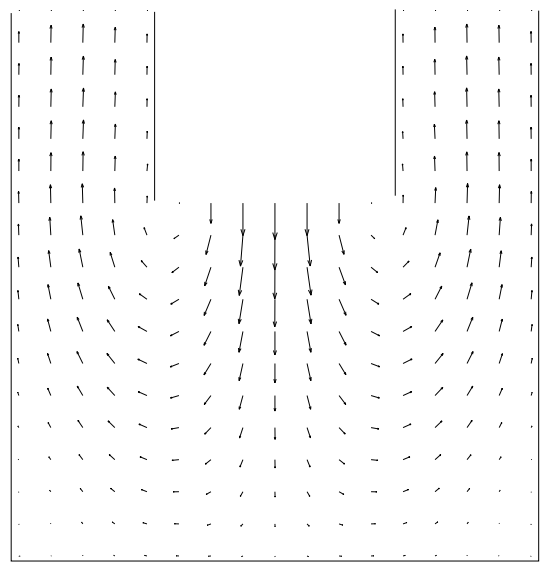

b) Controlled flow for $G r / R e^{2}=.9$ 


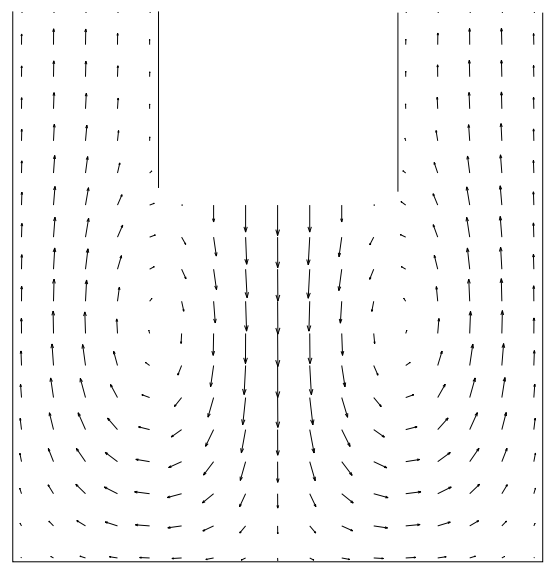

FIG. 11a) Uncontrolled flow for $G r / R e^{2}=1$

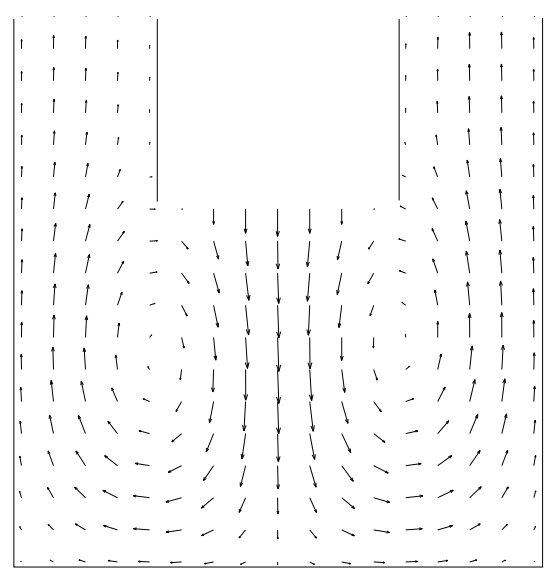

FIG. 12a) Uncontrolled flow for $G r / R e^{2}=1.1$

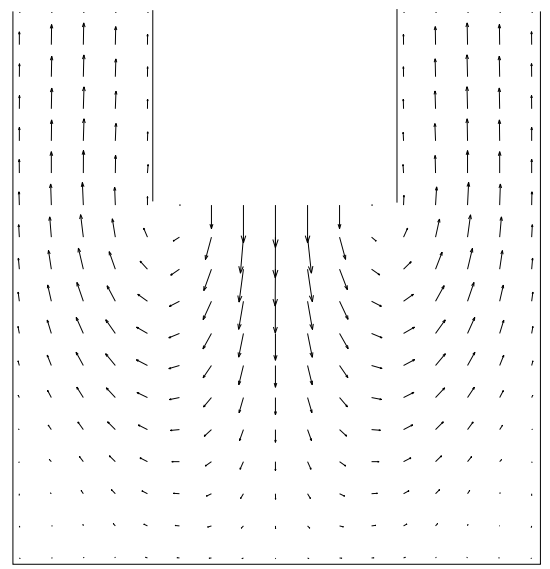

b) Controlled flow for $G r / R e^{2}=1$

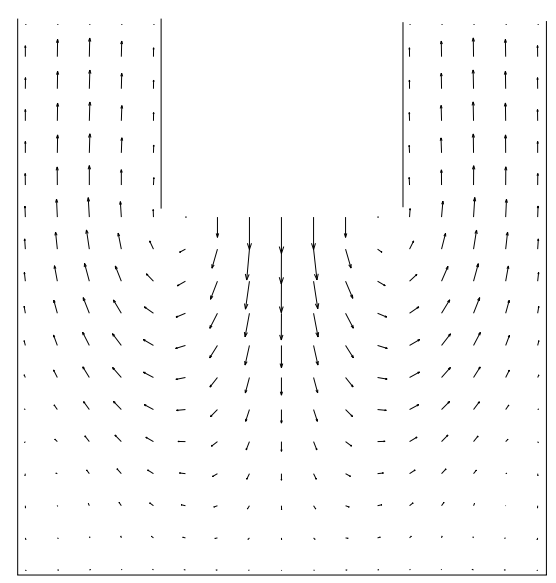

b) Controlled flow for $G r / R e^{2}=1.1$ 


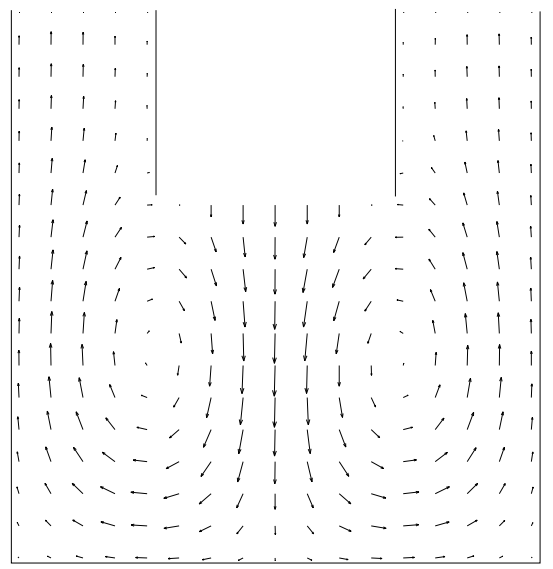

FIG. 13a) Uncontrolled flow for $G r / R e^{2}=1.2$

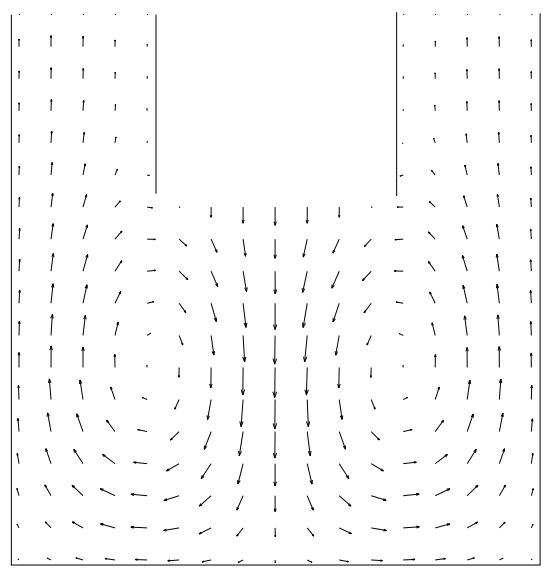

FIG. 14a) Uncontrolled flow for $G r / R e^{2}=1.3$

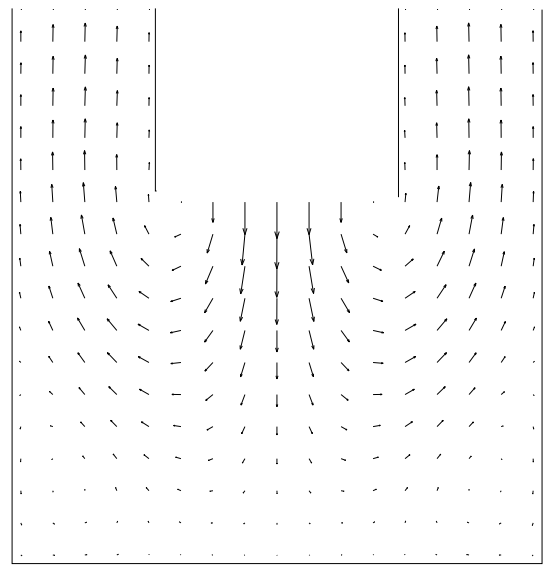

b) Controlled flow for $G r / R \epsilon^{2}=1.2$

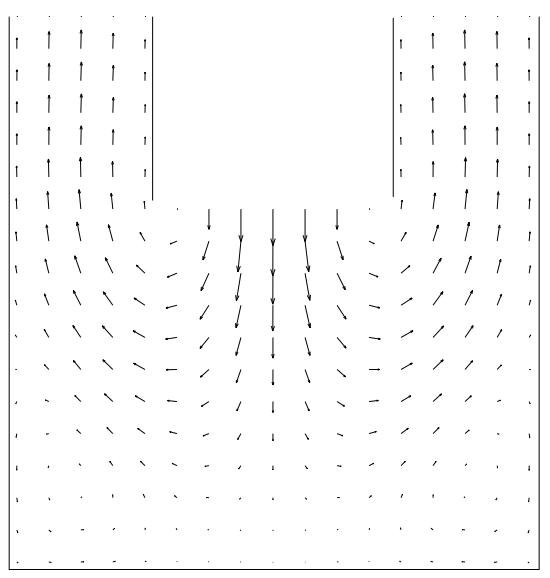

b) Controlled flow for $G r / R \epsilon^{2}=1.3$ 


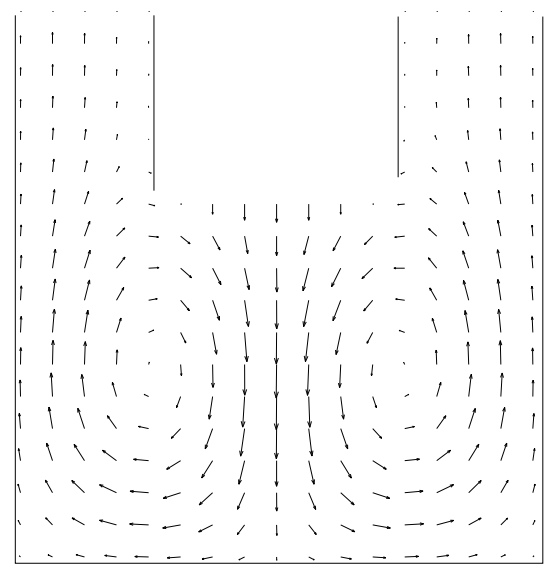

FIG. 15a) Uncontrolled flow for $G r / R e^{2}=1.4$

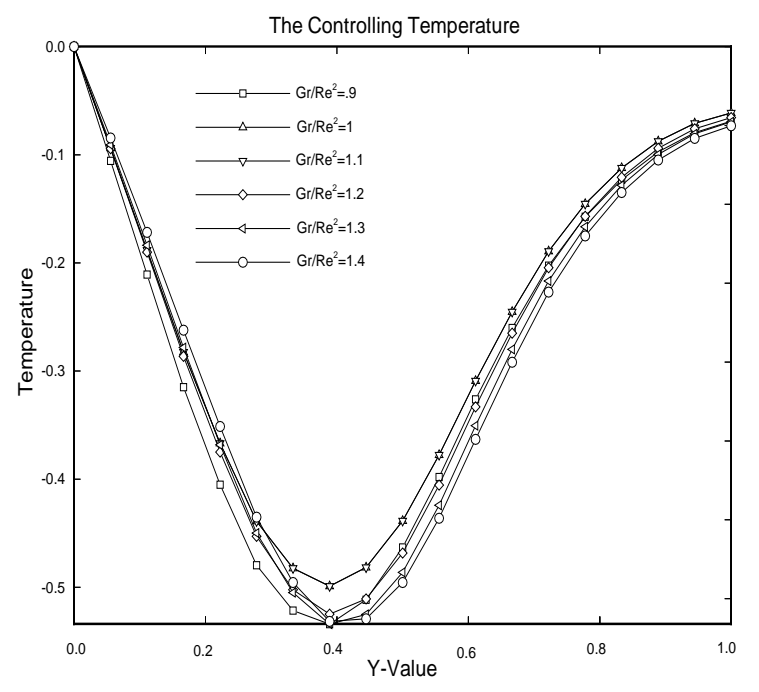

FIG. 16a) Control distribution along $\mathrm{x}=0$

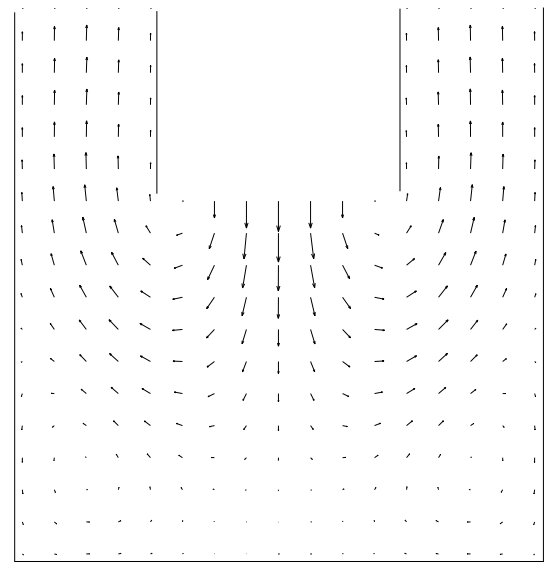

b) Controlled flow for $G r / R e^{2}=1.4$

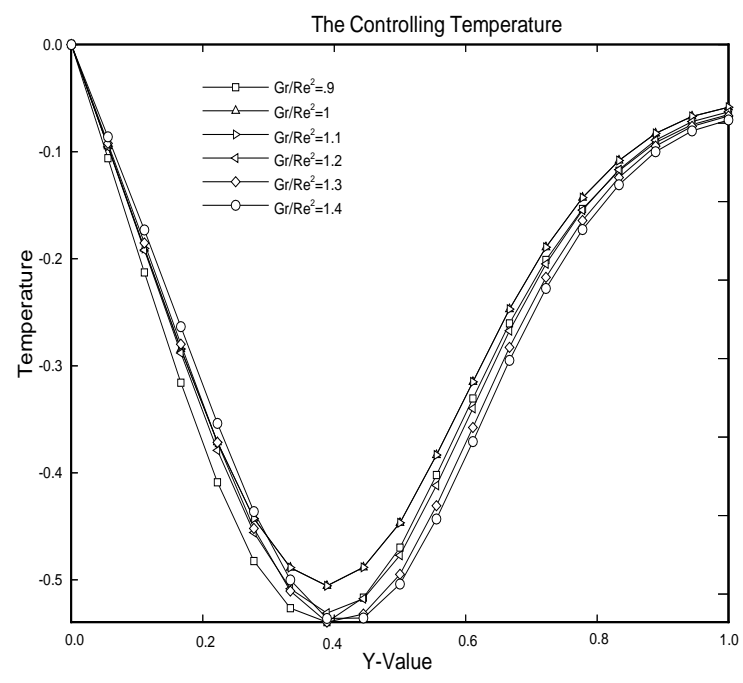

b) Control distribution along $x=1$. 